\title{
On Bayesian Asymptotics in Stochastic Differential Equations with Random Effects
}

\author{
Trisha Maitra and Sourabh Bhattacharya*
}

\begin{abstract}
Delattre et al. (2013) investigated asymptotic properties of the maximum likelihood estimator of the population parameters of the random effects associated with $n$ independent stochastic differential equations ( $S D E$ 's) assuming that the $S D E$ 's are independent and identical ( $i i d)$.

In this article, we consider the Bayesian approach to learning about the population parameters, and prove consistency and asymptotic normality of the corresponding posterior distribution in the iid set-up as well as when the $S D E$ 's are independent but non-identical.
\end{abstract}

Keywords: Asymptotic normality; Maximum likelihood estimator; Posterior consistency; Posterior normality; Random effects; Stochastic differential equations.

\section{Introduction}

Mixed effects models are appropriate when dealing with data sets consisting of variability between subjects and also within subjects, with respect to time. Although a great deal of work on mixed effects models exists in the statistical literature, mixed effects models where within subject variability is modeled via stochastic differential equations ( $S D E$ 's) are relatively rare. For a relatively short but comprehensive review we refer the reader to Delattre et al. (2013), who also undertake theoretical and asymptotic investigation of a class of $S D E$-based mixed effects models having the following form: for $i=1, \ldots, n$,

$$
d X_{i}(t)=b\left(X_{i}(t), \phi_{i}\right) d t+\sigma\left(X_{i}(t)\right) d W_{i}(t),
$$

where, for $i=1, \ldots, n, X_{i}(0)=x^{i}$ is the initial value of the stochastic process $X_{i}(t)$, which is assumed to be continuously observed on the time interval $\left[0, T_{i}\right] ; T_{i}>0$ assumed to be known. The function $b(x, \varphi)$ is a known, real-valued function on $\mathbb{R} \times \mathbb{R}^{d}$ ( $\mathbb{R}$ is the real line and $d$ is the dimension); this function is known as the drift function. The function $\sigma: \mathbb{R} \mapsto \mathbb{R}$ is the known diffusion coefficient. In the context of statistical modelling, $X_{i}(\cdot)$ models the $i$-th individual. The $S D E$ 's given by (1.1) are driven by independent standard Wiener processes $\left\{W_{i}(\cdot) ; i=1, \ldots, n\right\}$, and $\left\{\phi_{i} ; i=1, \ldots, n\right\}$, which are to be interpreted as the random effect parameters associated with the $n$ individuals, are assumed to be independent of the Brownian motions and independently and identically distributed (iid) random variables with common distribution $g(\varphi, \theta) d \nu(\varphi)$. Here $g(\varphi, \theta)$ is a density with respect to a dominating measure on $\mathbb{R}^{d}$, for all $\theta$, where $\theta \in \Omega \subset \mathbb{R}^{p}(p \geq 2 d)$ is the unknown parameter of interest, which is to be estimated. Delattre et al. (2013) impose regularity conditions that ensure existence of solutions of (1.1). The conditions, which are also adopted by us, are as follows.

(H1) (i) The function $(x, \varphi) \mapsto b(x, \varphi)$ is $C^{1}$ (differentiable with continuous first derivative) on $\mathbb{R} \times \mathbb{R}^{d}$, and such that there exists $K>0$ so that

$$
b^{2}(x, \varphi) \leq K\left(1+x^{2}+|\varphi|^{2}\right),
$$

for all $(x, \varphi) \in \mathbb{R} \times \mathbb{R}^{d}$.

(ii) The function $\sigma(\cdot)$ is $C^{1}$ on $\mathbb{R}$ and

$$
\sigma^{2}(x) \leq K\left(1+x^{2}\right),
$$

for all $x \in \mathbb{R}$.

\footnotetext{
${ }^{*}$ Trisha Maitra is a PhD student and Sourabh Bhattacharya is an Assistant Professor in Interdisciplinary Statistical Research
} Unit, Indian Statistical Institute, 203, B. T. Road, Kolkata 700108. Corresponding e-mail: sourabh@isical.ac.in. 
(H2) Let $X_{i}^{\varphi}$ be associated with the SDE of the form 11.1 with drift function $b(x, \varphi)$. Also letting $Q_{\varphi}^{x^{i}, T_{i}}$ denote the joint distribution of $\left\{X_{i}^{\varphi}(t) ; t \in\left[0, T_{i}\right]\right\}$, it is assumed that for $i=1, \ldots, n$, and for all $\varphi, \varphi^{\prime}$, the following holds:

$$
Q_{\varphi}^{x^{i}, T_{i}}\left(\int_{0}^{T_{i}} \frac{b^{2}\left(X_{i}^{\varphi}(t), \varphi^{\prime}\right)}{\sigma^{2}\left(X_{i}^{\varphi}(t)\right)} d t<\infty\right)=1
$$

(H3) For $f=\frac{\partial b}{\partial \varphi_{j}}, j=1, \ldots, d$, there exist $c>0$ and some $\gamma \geq 0$ such that

$$
\sup _{\varphi \in \mathbb{R}^{d}} \frac{|f(x, \varphi)|}{\sigma^{2}(x)} \leq c\left(1+|x|^{\gamma}\right)
$$

In this article, we consider $d=1$, that is, we assume one-dimensional random effects, so that $\varphi \in \mathbb{R}$. Moreover, as in Delattre et al. (2013), for statistical inference we assume that $b\left(x, \phi_{i}\right)$ is linear in $\phi_{i}$; in other words, $b\left(x, \phi_{i}\right)=\phi_{i} b(x)$. Under this assumption, (H3) is not required; see Delattre et al. (2013) and Maitra and Bhattacharya (2016). Following Maitra and Bhattacharya (2016) we further assume that

$\left(\mathrm{H}^{\prime}\right) \quad b(\cdot)$ and $\sigma(x)$ are $C^{1}$ on $\mathbb{R}$ satisfying $b^{2}(x) \leq K\left(1+x^{2}\right)$ and $\sigma^{2}(x) \leq K\left(1+x^{2}\right)$ for all $x \in \mathbb{R}$, for some $K>0$.

(H2') Almost surely for each $i \geq 1$,

$$
\int_{0}^{T_{i}} \frac{b^{2}\left(X_{i}(s)\right)}{\sigma^{2}\left(X_{i}(s)\right)} d s<\infty
$$

As in Delattre et al. (2013) and Maitra and Bhattacharya (2016) here we assume that $\phi_{i}$ are normally distributed implying for $k \geq 1, E\left|\phi_{i}\right|^{2 k}<\infty$ so that the following holds for all $T>0$ (see Delattre et al. (2013)):

$$
\sup _{t \in[0, T]} E\left[X_{i}(t)\right]^{2 k}<\infty
$$

In fact, the linearity assumption $b\left(x, \phi_{i}\right)=\phi_{i} b(x)$ and the assumption that $\phi_{i}$ are Gaussian random variables are crucial for availability of an explicit form of the likelihood of the parameters of the random effects $\phi_{i}$. Indeed, assuming that $g(\varphi, \theta) d \nu(\varphi) \equiv N\left(\mu, \omega^{2}\right)$, Delattre et al. (2013) obtain the likelihood as the product of the following:

$$
f_{i}\left(X_{i} \mid \theta\right)=\frac{1}{\left(1+\omega^{2} V_{i}\right)^{1 / 2}} \exp \left[-\frac{V_{i}}{2\left(1+\omega^{2} V_{i}\right)}\left(\mu-\frac{U_{i}}{V_{i}}\right)^{2}\right] \exp \left(\frac{U_{i}^{2}}{2 V_{i}}\right),
$$

where $\theta=\left(\mu, \omega^{2}\right) \in \mathbb{R} \times \mathbb{R}^{+}\left(R^{+}=(0, \infty)\right)$, and

$$
U_{i}=\int_{0}^{T_{i}} \frac{b\left(X_{i}(s)\right)}{\sigma^{2}\left(X_{i}(s)\right)} d X_{i}(s), \quad V_{i}=\int_{0}^{T_{i}} \frac{b^{2}\left(X_{i}(s)\right)}{\sigma^{2}\left(X_{i}(s)\right)} d s ; \quad i=1, \ldots, n,
$$

are sufficient statistics. In $\left[1.3\right.$, for $i=1, \ldots, n, X_{i}$ stands for $\left\{X_{i}(t) ; t \in\left[0, T_{i}\right]\right\}$.

Delattre et al. (2013) consider the $i i d$ set-up by setting $x^{i}=x$ and $T_{i}=T$ for $i=1, \ldots, n$, and directly prove weak consistency (convergence in probability) and asymptotic normality of the $M L E$ of $\theta$. As an alternative, Maitra and Bhattacharya (2016) verify the regularity conditions of existing results in general set-ups provided in Schervish (1995) and Hoadley (1971) to prove asymptotic properties of the $M L E$ in this $S D E$ set-up. In the $i i d$ set-up, this approach allowed Maitra and Bhattacharya (2016) to establish strong consistency of the $M L E$, rather than weak consistency. Moreover, assumption (H4) of Delattre et al. (2013), requiring that $b(\cdot) / \sigma(\cdot)$ is non-constant and for $i \geq 1,\left(U_{i}, V_{i}\right)$ admits a density with respect to the Lebesgue measure on $\mathbb{R} \times \mathbb{R}^{+}$which is jointly continuous and positive on an open ball of $\mathbb{R} \times \mathbb{R}^{+}$, was not required in their approach. Also, not only in the iid situation, Maitra and 
Bhattacharya (2016) prove asymptotic results related to the $M L E$ even in the independent but nonidentical (we refer to this as non-iid) case.

To our knowledge, Bayesian asymptotics has not been investigated in the context of mixed effects models, even though applied Bayesian analysis of such models is not rare (see, for example, Wakefield et al. (1994), Wakefield (1996), Bennett et al. (1996)). In this article, we consider the Bayesian framework associated with $S D E$-based random effects model, for both iid and non-iid set-ups, and prove consistency and asymptotic normality of the Bayesian posterior distribution of $\theta=\left(\mu, \omega^{2}\right)$. In other words, we consider prior distributions $\pi(\theta)$ of $\theta$ and study the properties of the corresponding posterior

$$
\pi_{n}\left(\theta \mid X_{1}, \ldots, X_{n}\right)=\frac{\pi(\theta) \prod_{i=1}^{n} f_{i}\left(X_{i} \mid \theta\right)}{\int_{\psi \in \Omega} \pi(\psi) \prod_{i=1}^{n} f_{i}\left(X_{i} \mid \psi\right) d \psi}
$$

as the sample size $n$ tends to infinity. In what follows, in Section 2 we investigate asymptotic properties of the posterior in the iid context. In Section 3 we investigate Bayesian asymptotics in the non-iid set-up. We summarize our contribution and provide further discussion in Section 4. Further details are provided in the supplement Maitra and Bhattacharya (2015), whose sections, tables and figures have the prefix "S-" when referred to in this paper. Indeed in Section S-1 of the supplement we illustrate with examples when posterior consistency holds and fails; in the same section we also include examples pertaining to consistency and inconsistency in a dependent set-up, which provide insights regarding extension of our asymptotic theory to dependent sets of $S D E$ 's. In Section S-2 of the supplement we illustrate with an example the advantages of the Bayesian inference over classical studies in $S D E$-based random effects models. In Section S-3 we provide a brief discussion on choice of prior and associated posterior computations in practical applications.

For the purpose of asymptotics, we adopt two further assumptions of Delattre et al. (2013) (also adopted by Maitra and Bhattacharya (2016)), given by

$\left(\mathrm{H} 3^{\prime}\right)$ The parameter space $\Omega$ is a compact subset of $\mathbb{R} \times \mathbb{R}^{+}$.

$\left(\mathrm{H} 4^{\prime}\right)$ The true value $\theta_{0} \in \Omega$.

Recall that condition (H4) of Delattre et al. (2013) was not required by Maitra and Bhattacharya (2016) in their classical approach. Neither is the assumption required in our Bayesian approach. Also, as we shall show, our condition $\left(\mathrm{H}^{\prime}\right)$ on compactness of $\Omega$, is not necessary for posterior consistency in the non-iid case. Notationally, “a.s., " “ $\stackrel{P}{\rightarrow}$ ” and $\stackrel{\mathcal{L}}{\rightarrow}$ ” denote convergence "almost surely", "in probability" and "in distribution", respectively.

\section{Consistency and asymptotic normality of the Bayesian posterior in the iid set-up}

\subsection{Consistency of the Bayesian posterior distribution}

Theorem 7.80 presented in Schervish (1995) provides easy-to-verify sufficient conditions that ensure posterior consistency. We state the general theorem below, using which we prove posterior consistency in our case.

Theorem 1 (Schervish (1995)) Let $\left\{X_{n}\right\}_{n=1}^{\infty}$ be conditionally iid given $\theta$ with density $f_{1}(x \mid \theta)$ with respect to a measure $\nu$ on a space $\left(\mathcal{X}^{1}, \mathcal{B}^{1}\right)$. Fix $\theta_{0} \in \Omega$, and define, for each $M \subseteq \Omega$ and $x \in \mathcal{X}^{1}$,

$$
Z(M, x)=\inf _{\psi \in M} \log \frac{f_{1}\left(x \mid \theta_{0}\right)}{f_{1}(x \mid \psi)} .
$$

Assume that for each $\theta \neq \theta_{0}$, there is an open set $N_{\theta}$ such that $\theta \in N_{\theta}$ and that $E_{\theta_{0}} Z\left(N_{\theta}, X_{i}\right)>-\infty$. Also assume that $f_{1}(x \mid \cdot)$ is continuous at $\theta$ for every $\theta$, a.s. $\left[P_{\theta_{0}}\right]$. For $\epsilon>0$, define $C_{\epsilon}=\{\theta$ : 
$\left.\mathcal{K}_{1}\left(\theta_{0}, \theta\right)<\epsilon\right\}$, where

$$
\mathcal{K}_{1}\left(\theta_{0}, \theta\right)=E_{\theta_{0}}\left(\log \frac{f_{1}\left(X_{1} \mid \theta_{0}\right)}{f_{1}\left(X_{1} \mid \theta\right)}\right)
$$

is the Kullback-Leibler divergence measure associated with observation $X_{1}$. Let $\pi$ be a prior distribution such that $\pi\left(C_{\epsilon}\right)>0$, for every $\epsilon>0$. Then, for every $\epsilon>0$ and open set $\mathcal{N}_{0}$ containing $C_{\epsilon}$, the posterior satisfies

$$
\lim _{n \rightarrow \infty} \pi_{n}\left(\mathcal{N}_{0} \mid X_{1}, \ldots, X_{n}\right)=1, \quad \text { a.s. } \quad\left[P_{\theta_{0}}\right] .
$$

\subsubsection{Verification of posterior consistency}

The condition $E_{\theta_{0}} Z\left(N_{\theta}, X_{i}\right)>-\infty$ of the above theorem is verified in the context of Theorem 1 in Maitra and Bhattacharya (2016). Here we provide a briefing on this. Note that in our case $f_{1}(x \mid \theta)$ is given by (1.3). It then follows from the proof of Proposition 7 of Delattre et al. (2013) that for every $\theta \neq \theta_{0}$,

$$
\begin{aligned}
\log \frac{f_{1}\left(x \mid \theta_{0}\right)}{f_{1}(x \mid \theta)} \geq & -\frac{1}{2}\left\{\log \left(1+\frac{\omega^{2}}{\omega_{0}^{2}}\right)+\frac{\left|\omega^{2}-\omega_{0}^{2}\right|}{\omega^{2}}\right\}-\frac{1}{2}\left|\omega_{0}^{2}-\omega^{2}\right|\left(\frac{U_{1}}{1+\omega_{0}^{2} V_{1}}\right)^{2}\left(1+\frac{\omega_{0}^{2}}{\omega^{2}}\right) \\
& -|\mu|\left|\frac{U_{1}}{1+\omega_{0}^{2} V_{1}}\right|\left(1+\frac{\left|\omega_{0}^{2}-\omega^{2}\right|}{\omega^{2}}\right)-\left|\frac{\mu_{0}^{2} V_{1}}{2\left(1+\omega_{0}^{2} V_{1}\right)}\right|-\left|\frac{\mu_{0} U_{1}}{1+\omega_{0}^{2} V_{1}}\right| .
\end{aligned}
$$

Taking $N_{\theta}=(\underline{\mu}, \bar{\mu}) \times\left(\underline{\omega}^{2}, \bar{\omega}^{2}\right)$, and making use of Lemma 1 of Delattre et al. (2013) shows that $E_{\theta_{0}}\left(\frac{U_{1}}{1+\omega_{0}^{2} V_{1}}\right)^{2}, E_{\theta_{0}}\left|\frac{U_{1}}{1+\omega_{0}^{2} V_{1}}\right|$ and $E_{\theta_{0}}\left(\frac{\mu_{0}^{2} V_{1}}{2\left(1+\omega_{0}^{2} V_{1}\right)}\right)$ are finite. Hence, $E_{\theta_{0}} Z\left(N_{\theta}, X_{i}\right)>-\infty$.

Now, all we need to ensure is that there exists a prior $\pi$ which gives positive probability to $C_{\epsilon}$ for every $\epsilon>0$. From the identifiability result given by Proposition 7 (i) of Delattre et al. (2013) it follows that $\mathcal{K}_{1}\left(\theta_{0}, \theta\right)=0$ if and only if $\theta=\theta_{0}$. Hence, for any $\epsilon>0$, the set $C_{\epsilon}$ is non-empty, since it contains at least $\theta_{0}$. In fact, since Delattre et al. (2013) also show that $\mathcal{K}_{1}\left(\theta_{0}, \theta\right)$ is continuous in $\theta$ (Proposition 7 (ii)), and since the parameter space $\Omega$ is compact, it follows that $\mathcal{K}_{1}\left(\theta_{0}, \theta\right)$ is uniformly continuous on $\Omega$. Hence, for any $\epsilon>0$, there exists $\delta_{\epsilon}$ which is independent of $\theta$, such that $\left\|\theta-\theta_{0}\right\| \leq \delta_{\epsilon}$ implies $\mathcal{K}_{1}\left(\theta_{0}, \theta\right)<\epsilon$. In other words, $\left\{\theta:\left\|\theta-\theta_{0}\right\| \leq \delta_{\epsilon}\right\} \subseteq C_{\epsilon}$.

Let $\frac{d \pi}{d \nu}=h$ almost everywhere on $\Omega$, where $h(\theta)$ is any positive, continuous density on $\Omega$ with respect to the Lebesgue measure $\nu$. By "positive" density we mean a density excluding any interval of null measure. It then follows from the above arguments that

$$
\pi\left(C_{\epsilon}\right) \geq \pi\left(\left\{\theta:\left\|\theta-\theta_{0}\right\| \leq \delta_{\epsilon}\right\}\right) \geq\left[\inf _{\left\{\theta:\left\|\theta-\theta_{0}\right\| \leq \delta_{\epsilon}\right\}} h(\theta)\right] \times \nu\left(\left\{\theta:\left\|\theta-\theta_{0}\right\| \leq \delta_{\epsilon}\right\}\right)>0 .
$$

Hence, 2.2) holds in our case with any prior with positive, continuous density with respect to the Lebesgue measure. We summarize this result in the form of a theorem, stated below.

Theorem 2 Assume the iid set-up and conditions $\left(H 1^{\prime}\right)-\left(H 4^{\prime}\right)$. For $\epsilon>0$, define $C_{\epsilon}=\{\theta$ : $\left.\mathcal{K}_{1}\left(\theta_{0}, \theta\right)<\epsilon\right\}$, where

$$
\begin{aligned}
\mathcal{K}_{1}\left(\theta_{0}, \theta\right)= & E_{\theta_{0}}\left(\log \frac{f_{1}\left(X_{1} \mid \theta_{0}\right)}{f_{1}\left(X_{1} \mid \theta\right)}\right) \\
= & E_{\theta_{0}}\left[\frac{1}{2} \log \left(\frac{1+\omega^{2} V_{1}}{1+\omega_{0}^{2} V_{1}}\right)+\frac{1}{2} \frac{\left(\omega_{0}^{2}-\omega^{2}\right) U_{1}^{2}}{\left(1+\omega^{2} V_{1}\right)\left(1+\omega_{0}^{2} V_{1}\right)}\right. \\
& \left.+\frac{\mu^{2} V_{1}}{2\left(1+\omega^{2} V_{1}\right)}-\frac{\mu U_{1}}{1+\omega^{2} V_{1}}-\left(\frac{\mu_{0}^{2} V_{1}}{2\left(1+\omega_{0}^{2} V_{1}\right)}-\frac{\mu_{0} U_{1}}{1+\omega_{0}^{2} V_{1}}\right)\right]
\end{aligned}
$$

is the Kullback-Leibler divergence measure associated with observation $X_{1}$. Let the prior distribution 
$\pi$ of the parameter $\theta$ satisfy $\frac{d \pi}{d \nu}=h$ almost everywhere on $\Omega$, where $h(\theta)$ is any positive, continuous density on $\Omega$ with respect to the Lebesgue measure $\nu$. Then the posterior (1.5) is consistent in the sense that for every $\epsilon>0$ and open set $\mathcal{N}_{0}$ containing $C_{\epsilon}$, the posterior satisfies

$$
\lim _{n \rightarrow \infty} \pi_{n}\left(\mathcal{N}_{0} \mid X_{1}, \ldots, X_{n}\right)=1, \quad \text { a.s. } \quad\left[P_{\theta_{0}}\right] .
$$

\subsection{Asymptotic normality of the Bayesian posterior distribution}

We now investigate asymptotic normality of posterior distributions in our $S D E$ set-up. For our purpose, we make use of Theorem 7.102 in conjunction with Theorem 7.89 provided in Schervish (1995). These theorems make use of seven regularity conditions, of which only the first four will be required for the iid set-up. Hence, in this iid context we state the four requisite conditions.

\subsubsection{Regularity conditions - iid case}

(1) The parameter space is $\Omega \subseteq \mathbb{R}^{p}$ for some finite $p$.

(2) $\theta_{0}$ is a point interior to $\Omega$.

(3) The prior distribution of $\theta$ has a density with respect to Lebesgue measure that is positive and continuous at $\theta_{0}$.

(4) There exists a neighborhood $\mathcal{N}_{0} \subseteq \Omega$ of $\theta_{0}$ on which $\ell_{n}(\theta)=\log f\left(X_{1}, \ldots, X_{n} \mid \theta\right)$ is twice continuously differentiable with respect to all co-ordinates of $\theta$, a.s. $\left[P_{\theta_{0}}\right]$.

Before proceeding to justify asymptotic normality of our posterior, we furnish the relevant theorem below (Theorem 7.102 of Schervish (1995)).

Theorem 3 (Schervish (1995)) Let $\left\{X_{n}\right\}_{n=1}^{\infty}$ be conditionally iid given $\theta$. Assume the above four regularity conditions; also assume that there exists $H_{r}(x, \theta)$ such that, for each $\theta_{0} \in \operatorname{int}(\Omega)$ and each $k, j$,

$$
\sup _{\left\|\theta-\theta_{0}\right\| \leq r}\left|\frac{\partial^{2}}{\partial \theta_{k} \partial \theta_{j}} \log f_{1}\left(x \mid \theta_{0}\right)-\frac{\partial^{2}}{\partial \theta_{k} \partial \theta_{j}} \log f_{1}(x \mid \theta)\right| \leq H_{r}\left(x, \theta_{0}\right)
$$

with

$$
\lim _{r \rightarrow 0} E_{\theta_{0}} H_{r}\left(X, \theta_{0}\right)=0 .
$$

Further suppose that the conditions of Theorem 1 hold, and that the Fisher's information matrix $\mathcal{I}\left(\theta_{0}\right)$ is positive definite. Now denoting by $\hat{\theta}_{n}$ the $M L E$ associated with $n$ observations, let

$$
\Sigma_{n}^{-1}=\left\{\begin{array}{cc}
-\ell_{n}^{\prime \prime}\left(\hat{\theta}_{n}\right) & \text { if the inverse and } \hat{\theta}_{n} \text { exist } \\
\mathbb{I}_{\tau} & \text { if not },
\end{array}\right.
$$

where for any $t$,

$$
\ell_{n}^{\prime \prime}(t)=\left(\left(\left.\frac{\partial^{2}}{\partial \theta_{i} \partial \theta_{j}} \ell_{n}(\theta)\right|_{\theta=t}\right)\right)
$$

and $\mathbb{I}_{\tau}$ is the identity matrix of order $\tau$. Thus, $\Sigma_{n}^{-1}$ is the observed Fisher's information matrix.

Letting $\Psi_{n}=\Sigma_{n}^{-1 / 2}\left(\theta-\hat{\theta}_{n}\right)$, for each compact subset $B$ of $\mathbb{R}^{\tau}$ and each $\epsilon>0$, the following holds:

$$
\lim _{n \rightarrow \infty} P_{\theta_{0}}\left(\sup _{\Psi_{n} \in B}\left|\pi_{n}\left(\Psi_{n} \mid X_{1}, \ldots, X_{n}\right)-\xi\left(\Psi_{n}\right)\right|>\epsilon\right)=0,
$$

where $\xi(\cdot)$ denotes the density of the standard normal distribution. 


\subsubsection{Verification of posterior normality}

We make the following assumption:

$\left(\mathrm{H}^{\prime}\right)$ The Fisher's information matrix $\mathcal{I}\left(\theta_{0}\right)$ is positive definite (see Delattre et al. (2013) for the form of $\mathcal{I}\left(\theta_{0}\right)$ ).

Now observe that the four regularity conditions in Section 2.2.1 trivially hold. The remaining conditions of Theorem 3 are verified in the context of Theorem 2 of Maitra and Bhattacharya (2016). Briefly, $\frac{\partial^{2}}{\partial \theta_{k} \partial \theta_{j}} \log f_{1}(x \mid \theta)$ is differentiable in $\theta=\left(\mu, \omega^{2}\right)$ and the derivative has finite expectation, which ensure (2.6) and 2.7). Hence, 2.10 holds in our SDE set-up. We summarize this result in the form of the following theorem.

Theorem 4 Assume the iid set-up and conditions $\left(H 1^{\prime}\right)-\left(H 5^{\prime}\right)$. Regarding $\left(H 3^{\prime}\right)$ we assume, in particular, that $\theta_{0} \in \operatorname{int}(\Omega)$. Let the prior distribution $\pi$ of the parameter $\theta$ satisfy $\frac{d \pi}{d \nu}=h$ almost everywhere on $\Omega$, where $h(\theta)$ is any density with respect to the Lebesgue measure $\nu$ which is positive and continuous at $\theta_{0}$. Then, letting $\Psi_{n}=\Sigma_{n}^{-1 / 2}\left(\theta-\hat{\theta}_{n}\right)$, for each compact subset $B$ of $\mathbb{R} \times \mathbb{R}^{+}$and each $\epsilon>0$, the following holds:

$$
\lim _{n \rightarrow \infty} P_{\theta_{0}}\left(\sup _{\Psi_{n} \in B}\left|\pi_{n}\left(\Psi_{n} \mid X_{1}, \ldots, X_{n}\right)-\xi\left(\Psi_{n}\right)\right|>\epsilon\right)=0 .
$$

\section{Consistency and asymptotic normality of the Bayesian posterior in the non-iid set-up}

In this section, as in Maitra and Bhattacharya (2016), we do not enforce the restrictions $T_{i}=T$ and $x^{i}=x$ for $i=1, \ldots, n$. Consequently, here we deal with the set-up where the processes $X_{i}(\cdot) ; i=$ $1, \ldots, n$, are independently, but not identically distributed. Following Maitra and Bhattacharya (2016), we assume the following:

(H6') The sequences $\left\{T_{1}, T_{2}, \ldots\right\}$ and $\left\{x^{1}, x^{2}, \ldots,\right\}$ are sequences in compact sets $\mathfrak{T}$ and $\mathfrak{X}$, respectively, so that there exist convergent subsequences with limits in $\mathfrak{T}$ and $\mathfrak{X}$. For notational convenience, we continue to denote the convergent subsequences as $\left\{T_{1}, T_{2}, \ldots\right\}$ and $\left\{x^{1}, x^{2}, \ldots\right\}$. Let us denote the limits by $T^{\infty}$ and $x^{\infty}$, where $T^{\infty} \in \mathfrak{T}$ and $x^{\infty} \in \mathfrak{X}$.

Following Maitra and Bhattacharya (2016), we denote the process associated with the initial value $x$ and time point $t$ as $X(t, x)$, so that $X\left(t, x^{i}\right)=X_{i}(t)$, and $X_{i}=\left\{X_{i}(t) ; t \in\left[0, T_{i}\right]\right\}$. We also denote by $\phi(x)$ the random effect parameter associated with the initial value $x$ such that $\phi\left(x^{i}\right)=\phi_{i}$. We assume the following condition with respect to $\phi(x)$ :

$\left(\mathrm{H} 7^{\prime}\right) \phi(x)$ is a real-valued, continuous function of $x$, and that for $k \geq 1, \sup _{x \in \mathfrak{X}} E[\phi(x)]^{2 k}<\infty$.

As in Proposition 1 of Delattre et al. (2013), assumption $\left(\mathrm{H}^{\prime}\right)$ implies that for any $T>0$,

$$
\sup _{t \in[0, T], x \in \mathfrak{X}} E[X(t, x)]^{2 k}<\infty .
$$

For $x \in \mathfrak{X}$ and $T \in \mathfrak{T}$, let

$$
\begin{aligned}
& U(x, T)=\int_{0}^{T} \frac{b(X(s, x))}{\sigma^{2}(X(s, x))} d X(s, x) ; \\
& V(x, T)=\int_{0}^{T} \frac{b^{2}(X(s, x))}{\sigma^{2}(X(s, x))} d s .
\end{aligned}
$$

For the non-iid set-up we let $U_{i}=U\left(x^{i}, T_{i}\right)$ and $V_{i}=V\left(x^{i}, T_{i}\right)$. As in Maitra and Bhattacharya (2016) we further assume that 
$\left(\mathrm{H} 8^{\prime}\right)$

$$
\frac{b^{2}(x)}{\sigma^{2}(x)}<K\left(1+x^{\tau}\right), \text { for some } \tau \geq 1
$$

This assumption ensures that moments of all orders of $V(x, T)$ are finite. Then, by Theorem 3 of Maitra and Bhattacharya (2016), the moments of uniformly integrable continuous functions of $U(x, T)$, $V(x, T)$ and $\theta$ are continuous in $x, T$ and $\theta$. In particular, the Kullback-Leibler distance and the information matrix, which we denote by $\mathcal{K}_{x, T}\left(\theta_{0}, \theta\right)$ (or, $\mathcal{K}_{x, T}\left(\theta, \theta_{0}\right)$ ) and $\mathcal{I}_{x, T}(\theta)$ respectively to emphasize dependence on the initial values $x$ and $T$, are continuous in $x, T$ and $\theta$. For $x=x^{k}$ and $T=T_{k}$, if we denote the Kullback-Leibler distance and the Fisher's information as $\mathcal{K}_{k}\left(\theta_{0}, \theta\right)\left(\mathcal{K}_{k}\left(\theta, \theta_{0}\right)\right)$ and $\mathcal{I}_{k}(\theta)$, respectively, then continuity of $\mathcal{K}_{x, T}\left(\theta_{0}, \theta\right)$ (or $\mathcal{K}_{x, T}\left(\theta, \theta_{0}\right)$ ) and $\mathcal{I}_{x, T}\left(\theta_{0}\right)$ with respect to $x$ and $T$ ensures that as $x^{k} \rightarrow x^{\infty}$ and $T_{k} \rightarrow T^{\infty}, \mathcal{K}_{x^{k}, T_{k}}\left(\theta_{0}, \theta\right) \rightarrow \mathcal{K}_{x^{\infty}, T^{\infty}}\left(\theta_{0}, \theta\right)=\mathcal{K}\left(\theta_{0}, \theta\right)$, say. Similarly, $\mathcal{K}_{x^{k}, T_{k}}\left(\theta, \theta_{0}\right) \rightarrow \mathcal{K}\left(\theta, \theta_{0}\right)$ and $\mathcal{I}_{x^{k}, T_{k}}(\theta) \rightarrow \mathcal{I}_{x^{\infty}, T^{\infty}}(\theta)=\mathcal{I}(\theta)$, say. Thanks to compactness, the limits $\mathcal{K}\left(\theta_{0}, \theta\right), \mathcal{K}\left(\theta, \theta_{0}\right)$ and $\mathcal{I}(\theta)$ are well-defined Kullback-Leibler divergences and Fisher's information, respectively. Consequently (see Maitra and Bhattacharya (2016)), the following hold for any $\theta \in \Omega$,

$$
\begin{aligned}
\lim _{n \rightarrow \infty} \frac{\sum_{k=1}^{n} \mathcal{K}_{k}\left(\theta_{0}, \theta\right)}{n} & =\mathcal{K}\left(\theta_{0}, \theta\right) ; \\
\lim _{n \rightarrow \infty} \frac{\sum_{k=1}^{n} \mathcal{K}_{k}\left(\theta, \theta_{0}\right)}{n} & =\mathcal{K}\left(\theta, \theta_{0}\right) ; \\
\lim _{n \rightarrow \infty} \frac{\sum_{k=1}^{n} \mathcal{I}_{k}(\theta)}{n} & =\mathcal{I}(\theta) .
\end{aligned}
$$

We assume that

$\left(\mathrm{H} 9^{\prime}\right)$ For any $\theta \in \Omega, \mathcal{I}(\theta)$ is positive definite.

The above results will be seen to have important roles as we proceed with the non-iid Bayesian set-up. For consistency in the Bayesian framework we utilize the theorem of Choi and Schervish (2007), and for asymptotic normality of the posterior we make use of Theorem 7.89 of Schervish (1995).

\subsection{Posterior consistency in the non-iid set-up}

In our proceedings we need to ensure existence of moments of the form

$$
\sup _{x \in \mathcal{X}, T \in \mathfrak{T}} E_{\theta}\left[\exp \left\{\alpha\left|\omega_{0}^{2}-\omega^{2}\right|\left(\frac{U(x, T)}{1+\omega_{0}^{2} V(x, T)}\right)^{2}\left(1+\frac{\omega_{0}^{2}}{\omega^{2}}\right)\right\}\right],
$$

for some $0<\alpha<\infty$. The following extra assumption will be useful in this regard.

$\left(\mathrm{H} 10^{\prime}\right)$ There exists a strictly positive function $\alpha^{*}(x, T, \theta)$, continuous in $(x, T, \theta)$, such that for any $(x, T, \theta)$,

$$
E_{\theta}\left[\exp \left\{\alpha^{*}(x, T, \theta) K_{1} U^{2}(x, T)\right\}\right]<\infty,
$$

where $K_{1}=\sup _{\omega: \theta \in \Omega}\left|\omega_{0}^{2}-\omega^{2}\right|\left(1+\frac{\omega_{0}^{2}}{\omega^{2}}\right)$.

Now, let

$$
\alpha_{\min }^{*}=\inf _{x \in \mathfrak{X}, T \in \mathfrak{T}, \theta \in \Omega} \alpha^{*}(x, T, \theta),
$$

and

$$
\alpha=\min \left\{\alpha_{\min }^{*}, c^{*}\right\}
$$

where $0<c^{*}<1 / 16$.

Compactness ensures that $\alpha_{\min }^{*}>0$, so that $0<\alpha<1 / 16$. It also holds due to compactness that for $\theta \in \Omega$,

$$
\sup _{x \in \mathfrak{X}, T \in \mathfrak{T}} E_{\theta}\left[\exp \left\{\alpha K_{1} U^{2}(x, T)\right\}\right]<\infty .
$$


This ensures that

$$
\begin{aligned}
& \sup _{x \in \mathcal{X}, T \in \mathfrak{T}} E_{\theta}\left[\exp \left\{\alpha\left|\omega_{0}^{2}-\omega^{2}\right|\left(\frac{U(x, T)}{1+\omega_{0}^{2} V(x, T)}\right)^{2}\left(1+\frac{\omega_{0}^{2}}{\omega^{2}}\right)\right\}\right] \\
& \leq \sup _{x \in \mathfrak{X}, T \in \mathfrak{T}} E_{\theta}\left[\exp \left\{\alpha K_{1} U^{2}(x, T)\right\}\right] \\
& <\infty .
\end{aligned}
$$

This choice of $\alpha$ ensuring (3.10) will be useful in verification of the conditions of Theorem 5 , which we next state.

Theorem 5 (Choi and Schervish (2007)) Let $\left\{X_{i}\right\}_{i=1}^{\infty}$ be independently distributed with densities $\left\{f_{i}(\cdot \mid \theta)\right\}_{i=1}^{\infty}$, with respect to a common $\sigma$-finite measure, where $\theta \in \Omega$, a measurable space. The densities $f_{i}(\cdot \mid \theta)$ are assumed to be jointly measurable. Let $\theta_{0} \in \Omega$ and let $P_{\theta_{0}}$ be the joint distribution of $\left\{X_{i}\right\}_{i=1}^{\infty}$ when $\theta_{0}$ is the true value of $\theta$. Let $\left\{\Theta_{n}\right\}_{n=1}^{\infty}$ be a sequence of subsets of $\Omega$. Let $\theta$ have prior $\pi$ on $\Omega$. Define the following:

$$
\begin{aligned}
\Lambda_{i}\left(\theta_{0}, \theta\right) & =\log \frac{f_{i}\left(X_{i} \mid \theta_{0}\right)}{f_{i}\left(X_{i} \mid \theta\right)}, \\
\mathcal{K}_{i}\left(\theta_{0}, \theta\right) & =E_{\theta_{0}}\left(\Lambda_{i}\left(\theta_{0}, \theta\right)\right) \\
\varrho_{i}\left(\theta_{0}, \theta\right) & =\operatorname{Var}_{\theta_{0}}\left(\Lambda_{i}\left(\theta_{0}, \theta\right)\right) .
\end{aligned}
$$

Make the following assumptions:

(1) Suppose that there exists a set $B$ with $\pi(B)>0$ such that

(i) $\sum_{i=1}^{\infty} \frac{\underline{\varrho}_{i}\left(\theta_{0}, \theta\right)}{i^{2}}<\infty, \quad \forall \theta \in B$,

(ii) For all $\epsilon>0, \pi\left(B \cap\left\{\theta: \mathcal{K}_{i}\left(\theta_{0}, \theta\right)<\epsilon, \forall i\right\}\right)>0$.

(2) Suppose that there exist test functions $\left\{\Phi_{n}\right\}_{n=1}^{\infty}$, sets $\left\{\Omega_{n}\right\}_{n=1}^{\infty}$ and constants $C_{1}, C_{2}, c_{1}, c_{2}>0$ such that

(i) $\sum_{n=1}^{\infty} E_{\theta_{0}} \Phi_{n}<\infty$,

(ii) $\sup _{\theta \in \Theta_{n}^{c} \cap \Omega_{n}} E_{\theta}\left(1-\Phi_{n}\right) \leq C_{1} e^{-c_{1} n}$,

(iii) $\pi\left(\Omega_{n}^{c}\right) \leq C_{2} e^{-c_{2} n}$.

Then,

$$
\pi_{n}\left(\theta \in \Theta_{n}^{c} \mid X_{1}, \ldots, X_{n}\right) \rightarrow 0 \quad \text { a.s. }\left[P_{\theta_{0}}\right]
$$

\subsubsection{Validation of posterior consistency}

Recall that $f_{i}\left(X_{i} \mid \theta\right)$ in our case is given by $(1.3)$. From the proof of Proposition 7 of Delattre et al. 2013) it follows that $\left|\log \frac{f_{i}\left(X_{i} \mid \theta_{0}\right)}{f_{i}\left(X_{i} \mid \theta\right)}\right|$ has an upper bound which has finite expectation and square of expectation under $\theta_{0}$, and is uniform for all $\theta \in B$, where $B$ is of the form $[\mu, \bar{\mu}] \times\left[\underline{\omega}^{2}, \bar{\omega}^{2}\right]$, say, with $\mu<\bar{\mu}$ and $0<\underline{\omega}^{2}<\bar{\omega}^{2}$. Hence, for each $i, \varrho_{i}\left(\theta_{0}, \theta\right)$ is finite. Moreover, since the sequences $\left\{T_{1}, \bar{T}_{2}, \ldots\right\}$ and $\left\{x^{1}, x^{2}, \ldots\right\}$ belong to compact spaces $\mathfrak{T}$ and $\mathfrak{X}$, and the variance function $\varrho_{x, T}\left(\theta_{0}, \theta\right)$ viewed as a function of $x$ and $T$, is bounded by a function continuous in $x$ and $T, \varrho_{i}\left(\theta_{0}, \theta\right)<\kappa$, for some $0<\kappa<\infty$, uniformly in $i$. Continuity of $\varrho_{x, T}\left(\theta_{0}, \theta\right)$ follows as an application of Theorem 3 of Maitra and Bhattacharya (2016) where the required uniform integrability is assured by finiteness of the moments of all orders of the random variable $U(x, T) /\left\{1+\omega^{2} V(x, T)\right\}$, for every $x \in \mathfrak{X}, T \in \mathfrak{T}$ 
(follows from Lemma 1 of Delattre et al. (2013)) and compactness of $\mathfrak{X}$ and $\mathfrak{T}$. Hence, choosing a prior that gives positive probability to the set $B$, it follows that for all $\theta \in B$,

$$
\sum_{i=1}^{\infty} \frac{\varrho_{i}\left(\theta_{0}, \theta\right)}{i^{2}}<\kappa \sum_{i=1}^{\infty} \frac{1}{i^{2}}<\infty .
$$

Hence, condition (1)(i) holds.

To verify (1)(ii) note that because of compactness of $B, \mathcal{K}_{i}\left(\theta_{0}, \theta\right)$, which is continuous in $\theta$, is uniformly continuous in $B$. Hence, for every $\epsilon>0$, there exists $\delta_{i}(\epsilon)$ independent of $\theta$ such that $\left\|\theta-\theta_{0}\right\|<\delta_{i}(\epsilon)$ implies $\mathcal{K}_{i}\left(\theta_{0}, \theta\right)<\epsilon$. Let us define

$$
\delta(\epsilon)=\inf \left\{\delta_{x, T}(\epsilon): x \in \mathfrak{X}, T \in \mathfrak{T}\right\},
$$

where $\delta_{x, T}(\epsilon)$ is any strictly positive continuous function of $x$ and $T$, depending upon $\epsilon$ such that $\delta_{x^{i}, T_{i}}(\epsilon)=\delta_{i}(\epsilon)$, for every $i=1,2, \ldots$. Compactness of $\mathfrak{X}$ and $\mathfrak{T}$ ensures that $\delta(\epsilon)>0$. So, for any $\epsilon>0$,

$$
\left\{\theta \in B: \mathcal{K}_{i}\left(\theta_{0}, \theta\right)<\epsilon, \forall i\right\} \supseteq\left\{\theta \in B:\left\|\theta-\theta_{0}\right\|<\delta(\epsilon)\right\}
$$

It follows that

$$
\pi\left(B \cap\left\{\theta: \mathcal{K}_{i}\left(\theta_{0}, \theta\right)<\epsilon, \forall i\right\}\right) \geq \pi\left(\left\{\theta \in B:\left\|\theta-\theta_{0}\right\|<\delta(\epsilon)\right\}\right) .
$$

The remaining part of the proof that the right hand side of $(3.15)$ is strictly positive, follows exactly in the same way as the proof of strict positivity 2.3 in Section 2.1.1, with a positive, continuous prior density on $\Omega$ with respect to the Lebesgue measure.

We now verify conditions (2)(i), (2)(ii) and (2)(iii). We let $\Omega_{n}=\left(\Omega_{1 n} \times \mathbb{R}^{+}\right)$, where $\Omega_{1 n}=$ $\left\{\mu:|\mu|<M_{n}\right\}$, where $M_{n}=O\left(e^{n}\right)$. Note that

$$
\pi\left(\Omega_{n}^{c}\right)=\pi\left(\Omega_{1 n}^{c}\right)=\pi\left(|\mu|>M_{n}\right)<E_{\pi}(|\mu|) M_{n}^{-1},
$$

so that (2)(iii) holds, assuming that the prior $\pi$ is such that the expectation $E_{\pi}(|\mu|)$ is finite.

Fixing $\delta>0$, we construct the tests $\Phi_{n}$ as follows.

$$
\Phi_{n}=\left\{\begin{array}{ccc}
1 & \text { if } & \beta_{n}<\sqrt{e^{-n \delta}} \\
0 & \text { otherwise }
\end{array}\right.
$$

where

$$
\beta_{n}=\frac{L_{n}\left(\theta_{0}\right)}{\sup _{\theta \in \Omega} L_{n}(\theta)}=\frac{L_{n}\left(\theta_{0}\right)}{L_{n}\left(\hat{\theta}_{n}\right)}
$$

is the likelihood ratio test statistic under $H_{0}: \theta=\theta_{0}$ versus $H_{1}: \theta \neq \theta_{0}$. Here $L_{n}(\theta)=\prod_{i=1}^{n} f_{i}\left(X_{i} \mid \theta\right)$, and, as before, $\hat{\theta}_{n}$ is the $M L E$ associated with $n$ observations. Now, denoting $-2 \log \beta_{n}$ by $Z_{n}^{2}$, we obtain for $\alpha$ given by (3.9),

$$
E_{\theta_{0}} \Phi_{n}=P_{\theta_{0}}\left(\beta_{n}<\sqrt{e^{-n \delta}}\right)=P_{\theta_{0}}\left(Z_{n}^{2}>n \delta\right)<e^{-\alpha n \delta} E_{\theta_{0}}\left(e^{\alpha Z_{n}^{2}}\right) .
$$

Note that $Z_{n}^{2}=-n\left(\hat{\theta}_{n}-\theta_{0}\right)^{T} \frac{\ell_{n}^{\prime \prime}\left(\theta_{n}^{*}\right)}{n}\left(\hat{\theta}_{n}-\theta_{0}\right)$, where $\ell_{n}(\theta)=\sum_{i=1}^{n} \log f_{i}\left(X_{i} \mid \theta\right)$, and $\theta_{n}^{*}$ lies between $\theta_{0}$ and $\hat{\theta}_{n}$. Also,

$$
\frac{\ell_{n, i j}^{\prime \prime}\left(\theta_{n}^{*}\right)}{n}=\frac{\ell_{n, i j}^{\prime \prime}\left(\theta_{0}\right)}{n}+\left(\theta_{n}^{*}-\theta_{0}\right)^{T} \frac{\ell_{n, i j}^{\prime \prime \prime}\left(\theta_{n}^{* *}\right)}{n},
$$

where $\ell_{n, i j}^{\prime \prime}$ is the $(i, j)$-th element of $\ell_{n}^{\prime \prime}$ and $\ell_{n, i j}^{\prime \prime \prime}$ is its derivative, and $\theta_{n}^{* *}$ lies between $\theta_{0}$ and $\theta_{n}^{*}$. Using 
Kolmogorov's strong law of large numbers for the non-iid case (see, for example, Serfling (1980)), which holds in our problem due to finiteness of the moments of $U(x, T) /\left\{1+\omega^{2} V(x, T)\right\}$ for every $x$ and $T$ belonging to the compact spaces $\mathfrak{X}$ and $\mathfrak{T}$, respectively, yields, in conjunction with $(3.7)$, that

$$
\frac{\ell_{n, i j}^{\prime \prime}\left(\theta_{0}\right)}{n} \stackrel{\text { a.s. }}{\rightarrow}-\mathcal{I}_{i j}\left(\theta_{0}\right),
$$

$\mathcal{I}_{i j}\left(\theta_{0}\right)$ being the $(i, j)$-th element of $\mathcal{I}\left(\theta_{0}\right)$. Also, by Cauchy-Schwartz,

$$
\left|\left(\theta_{n}^{*}-\theta_{0}\right)^{T} \frac{\ell_{n, i j}^{\prime \prime \prime}\left(\theta_{n}^{* *}\right)}{n}\right| \leq\left\|\theta_{n}^{*}-\theta_{0}\right\| \times\left\|\frac{\ell_{n, i j}^{\prime \prime \prime}\left(\theta_{n}^{* *}\right)}{n}\right\| .
$$

In (3.22), due to boundedness of the third derivative (see the proof of Proposition 8 of Delattre et al. (2013)), and due to continuity of the moments of $U(x, T) /\left\{1+\omega^{2} V(x, T)\right\}$ with respect to $x$ and $T$ (which follows from Theorem 3 of Maitra and Bhattacharya (2016) where uniform integrability is ensured by finiteness of the moments of the aforementioned function for every $x, T$ belonging to compact sets $\mathfrak{X}$ and $\mathfrak{T}$ ), and then finally applying Kolmogorov's strong law of large numbers for the non-iid case, it can be easily shown that $\left\|\frac{\ell_{n, i j}^{\prime \prime}\left(\theta_{n}^{* *}\right)}{n}\right\|=O_{P}(1)$. Since $\left\|\hat{\theta}_{n}-\theta_{0}\right\|=o_{P}(1)$, it follows that $\left\|\theta_{n}^{*}-\theta_{0}\right\|=o_{P}(1)$ as well. Hence,

$$
\left|\left(\theta_{n}^{*}-\theta_{0}\right)^{T} \frac{\ell_{n, i j}^{\prime \prime \prime}\left(\theta_{n}^{* *}\right)}{n}\right|=o_{P}(1),
$$

implying that

$$
\frac{\ell_{n}^{\prime \prime}\left(\theta_{n}^{*}\right)}{n} \stackrel{P}{\rightarrow}-\mathcal{I}\left(\theta_{0}\right)
$$

Hence, due to (3.24) and due to asymptotic normality of $M L E$ in our non-iid set-up addressed in Maitra and Bhattacharya (2016), under $P_{\theta_{0}}$,

$$
Z_{n}^{2}=-n\left(\hat{\theta}_{n}-\theta_{0}\right)^{T} \frac{\ell_{n}^{\prime \prime}\left(\theta_{n}^{*}\right)}{n}\left(\hat{\theta}_{n}-\theta_{0}\right) \stackrel{\mathcal{L}}{\rightarrow} \chi_{1}^{2},
$$

and so, by the continuous mapping theorem, $e^{\alpha Z_{n}^{2}} \stackrel{\mathcal{L}}{\rightarrow} e^{\alpha \chi_{1}^{2}}$. Moreover, using the form $Z_{n}^{2}=-2 \log \beta_{n}=$ $-2 \sum_{i=1}^{n}\left(\log f_{i}\left(X_{i} \mid \theta_{0}\right)-\log f_{i}\left(X_{i} \mid \hat{\theta}_{n}\right)\right)$, we can write

$$
\begin{aligned}
E_{\theta_{0}}\left(e^{\alpha Z_{n}^{2}}\right) & =E_{X_{1}, \ldots, X_{n} \mid \theta_{0}}\left(e^{\alpha Z_{n}^{2}}\right) \\
& =E_{X_{1}, \ldots, X_{n} \mid \theta_{0}}\left[\exp \left\{-2 \alpha \sum_{i=1}^{n}\left(\log f_{i}\left(X_{i} \mid \theta_{0}\right)-\log f_{i}\left(X_{i} \mid \hat{\theta}_{n}\right)\right)\right\}\right] \\
& =E_{\hat{\theta}_{n} \mid \theta_{0}} E_{X_{1}, \ldots, X_{n} \mid \theta_{0}, \hat{\theta}_{n}}\left[\exp \left\{-2 \alpha \sum_{i=1}^{n}\left(\log f_{i}\left(X_{i} \mid \theta_{0}\right)-\log f_{i}\left(X_{i} \mid \hat{\theta}_{n}\right)\right)\right\}\right] \\
& =E_{\hat{\theta}_{n} \mid \theta_{0}} \prod_{i=1}^{n} E_{X_{i} \mid \theta_{0}, \hat{\theta}_{n}}\left[\exp \left\{-2 \alpha\left(\log f_{i}\left(X_{i} \mid \theta_{0}\right)-\log f_{i}\left(X_{i} \mid \hat{\theta}_{n}\right)\right)\right\}\right] \\
& =E_{\hat{\theta}_{n} \mid \theta_{0}}\left[E_{n}\left(\hat{\theta}_{n}, \theta_{0}\right)\right], \text { (say), }
\end{aligned}
$$

where

$$
E_{n}\left(\hat{\theta}_{n}, \theta_{0}\right)=\prod_{i=1}^{n} E_{X_{i} \mid \theta_{0}, \hat{\theta}_{n}}\left[\exp \left\{-2 \alpha\left(\log f_{i}\left(X_{i} \mid \theta_{0}\right)-\log f_{i}\left(X_{i} \mid \hat{\theta}_{n}\right)\right)\right\}\right]
$$


It follows from the lower bound obtained in the proof of Proposition 7 of Delattre et al. (2013), that conditional on $\hat{\theta}_{n}=\zeta=\left(\mu, \omega^{2}\right), \log f_{i}\left(X_{i} \mid \theta_{0}\right)-\log f_{i}\left(X_{i} \mid \hat{\theta}_{n}\right) \geq C_{3}\left(U_{i}, V_{i}, \zeta\right)$, where

$$
\begin{aligned}
C_{3}\left(U_{i}, V_{i}, \zeta\right)= & -\frac{1}{2}\left\{\log \left(1+\frac{\omega^{2}}{\omega_{0}^{2}}\right)+\frac{\left|\omega^{2}-\omega_{0}^{2}\right|}{\omega^{2}}\right\}-\frac{1}{2}\left|\omega_{0}^{2}-\omega^{2}\right|\left(\frac{U_{i}}{1+\omega_{0}^{2} V_{i}}\right)^{2}\left(1+\frac{\omega_{0}^{2}}{\omega^{2}}\right) \\
& -|\mu|\left|\frac{U_{i}}{1+\omega_{0}^{2} V_{i}}\right|\left(1+\frac{\left|\omega_{0}^{2}-\omega^{2}\right|}{\omega^{2}}\right)-\left|\frac{\mu_{0}^{2} V_{i}}{2\left(1+\omega_{0}^{2} V_{i}\right)}\right|-\left|\frac{\mu_{0} U_{i}}{1+\omega_{0}^{2} V_{i}}\right| .
\end{aligned}
$$

Hence, for every given $n \geq 1$, due to the lower bound 3.29 and assumption $\left(\mathrm{H} 10^{\prime}\right)$, the latter implying (3.11,

$$
\begin{aligned}
E_{n}\left(\zeta, \theta_{0}\right) & =\prod_{i=1}^{n} E_{X_{i} \mid \theta_{0}, \hat{\theta}_{n}=\zeta}\left[\exp \left\{-2 \alpha\left(\log f_{i}\left(X_{i} \mid \theta_{0}\right)-\log f_{i}\left(X_{i} \mid \zeta\right)\right)\right\}\right] \\
& \leq \prod_{i=1}^{n} E_{X_{i} \mid \theta_{0}, \hat{\theta}_{n}=\zeta}\left[\exp \left\{-2 \alpha C_{3}\left(U_{i}, V_{i}, \zeta\right)\right\}\right] \\
& <\infty
\end{aligned}
$$

for any $\zeta \in \Omega$. Now, due to compactness of $\Omega, E_{n}\left(\zeta, \theta_{0}\right) \leq \sup _{\vartheta \in \Omega} E_{n}\left(\vartheta, \theta_{0}\right)<\infty$, for every given $n$, so that it follows from $3.27,3.28,3.29$ and 3.30 that $E_{\theta_{0}}\left(e^{\alpha Z_{n}^{2}}\right)=E_{\hat{\theta}_{n} \mid \theta_{0}}\left[E_{n}\left(\hat{\theta}_{n}, \theta_{0}\right)\right] \leq$ $\sup _{\vartheta \in \Omega} E_{n}\left(\vartheta, \theta_{0}\right)<\infty$, for any given $n$. So, for $n$ at most finite, $\vartheta \in \Omega$

$$
\sup _{n \text { at most finite }} E_{\theta_{0}}\left(e^{\alpha Z_{n}^{2}}\right)<\infty
$$

In our problem, for large enough $n$, at most the following case can occur: for any given $\epsilon>0$, there exists $N_{0}(\epsilon)$ such that $\left|E_{\theta_{0}}\left(e^{\alpha Z_{n}^{2}}\right)-E_{\theta_{0}}\left(e^{\alpha \chi_{1}^{2}}\right)\right|<\epsilon$ for $n \geq N_{0}(\epsilon)$, where $E_{\theta_{0}}\left(e^{\alpha \chi_{1}^{2}}\right)<\infty$. Combining this with (3.31) it follows that

$$
\sup _{n \geq 1} E_{\theta_{0}}\left(e^{\alpha Z_{n}^{2}}\right)<\infty
$$

Using this in conjunction with summation over (3.19), it is easily seen that condition (2)(i) holds.

Let us now verify condition (2)(ii). For our purpose, let us define $\Theta_{n}=\Theta_{\delta}=\left\{\left(\mu, \omega^{2}\right): \mathcal{K}\left(\theta, \theta_{0}\right)<\delta\right\}$, where $\mathcal{K}\left(\theta, \theta_{0}\right)$, defined as in 3.6 , is the proper Kullback-Leibler divergence. Thus, $\mathcal{K}\left(\theta, \theta_{0}\right)>0$ if 
and only if $\theta \neq \theta_{0}$. Now,

$$
\begin{aligned}
E_{\theta}\left(1-\Phi_{n}\right) & \\
= & P_{\theta}\left(\beta_{n}>\sqrt{e^{-n \delta}}\right)=P_{\theta}\left(-2 \log \beta_{n}<n \delta\right) \\
= & P_{\theta}\left(-n\left(\hat{\theta}_{n}-\theta\right)^{T} \frac{\ell_{n}^{\prime \prime}\left(\vartheta_{n}^{*}\right)}{n}\left(\hat{\theta}_{n}-\theta\right)+2 \ell_{n}\left(\theta_{0}\right)-2 \ell_{n}(\theta)-2\left(\hat{\theta}_{n}-\theta\right)^{T} \ell_{n}^{\prime}(\theta)>-n \delta\right) \\
& \left(\text { here } \vartheta_{n}^{*} \operatorname{lies} \text { between } \theta \text { and } \hat{\theta}_{n}\right) \\
< & e^{\alpha n \delta} E_{\theta}\left(\exp \left\{-\alpha n\left(\hat{\theta}_{n}-\theta\right)^{T} \frac{\ell_{n}^{\prime \prime}\left(\vartheta_{n}^{*}\right)}{n}\left(\hat{\theta}_{n}-\theta\right)+2 \alpha \ell_{n}\left(\theta_{0}\right)-2 \alpha \ell_{n}(\theta)-2 \alpha\left(\hat{\theta}_{n}-\theta\right)^{T} \ell_{n}^{\prime}(\theta)\right\}\right), \\
\leq & e^{\alpha n \delta} E_{\theta}\left(\exp \left\{-\alpha n\left(\hat{\theta}_{n}-\theta\right)^{T} \frac{\ell_{n}^{\prime \prime}\left(\vartheta_{n}^{*}\right)}{n}\left(\hat{\theta}_{n}-\theta\right)+2 \alpha \ell_{n}\left(\theta_{0}\right)-2 \alpha \ell_{n}(\theta)+2 \alpha\left|\left(\hat{\theta}_{n}-\theta\right)^{T} \ell_{n}^{\prime}(\theta)\right|\right\}\right) \\
\leq & e^{\alpha n \delta} \sqrt{E_{\theta}\left(\exp \left\{-2 \alpha n\left(\hat{\theta}_{n}-\theta\right)^{T} \frac{\ell_{n}^{\prime \prime}\left(\vartheta_{n}^{*}\right)}{n}\left(\hat{\theta}_{n}-\theta\right)+4 \alpha \ell_{n}\left(\theta_{0}\right)-4 \alpha \ell_{n}(\theta)\right\}\right)} \\
& \times \sqrt{E_{\theta}\left(\exp \left\{4 \alpha\left|\left(\hat{\theta}_{n}-\theta\right)^{T} \ell_{n}^{\prime}(\theta)\right|\right\}\right)} \quad \text { (using Cauchy-Schwartz inequality), }
\end{aligned}
$$

where $\alpha$ is given by (3.9). Now observe that

$$
\begin{gathered}
-n\left(\hat{\theta}_{n}-\theta\right)^{T} \frac{\ell_{n}^{\prime \prime}\left(\vartheta_{n}^{*}\right)}{n}\left(\hat{\theta}_{n}-\theta\right) \stackrel{\mathcal{L}}{\rightarrow} \chi_{1}^{2}, \\
\frac{\ell_{n}\left(\theta_{0}\right)-\ell_{n}(\theta)}{n} \stackrel{\text { a.s. }}{\rightarrow}-\mathcal{K}\left(\theta, \theta_{0}\right) .
\end{gathered}
$$

The aforementioned convergence result 3.36 is another application of Kolmogorov's strong law of large numbers in the non-iid case; as such, similar arguments used to justify (3.21) remain valid here. The Cauchy-Schwartz inequality entails

$$
\begin{aligned}
& \left|\left(\hat{\theta}_{n}-\theta\right)^{T} \ell_{n}^{\prime}(\theta)\right|=\left|\left(\hat{\theta}_{n}-\theta\right)^{T} \mathcal{I}(\theta)^{1 / 2} \mathcal{I}(\theta)^{-1 / 2} \ell_{n}^{\prime}(\theta)\right| \\
& \leq \sqrt{n\left(\hat{\theta}_{n}-\theta\right)^{T} \mathcal{I}(\theta)\left(\hat{\theta}_{n}-\theta\right)} \times \sqrt{n^{-1}\left\{\ell_{n}^{\prime}(\theta)\right\}^{T} \mathcal{I}^{-1}(\theta) \ell_{n}^{\prime}(\theta)},
\end{aligned}
$$

where

$$
n\left(\hat{\theta}_{n}-\theta\right)^{T} \mathcal{I}(\theta)\left(\hat{\theta}_{n}-\theta\right) \stackrel{\mathcal{L}}{\rightarrow} \chi_{1}^{2}
$$

and

$$
n^{-1}\left\{\ell_{n}^{\prime}(\theta)\right\}^{T} \mathcal{I}^{-1}(\theta) \ell_{n}^{\prime}(\theta)=n^{-1} \operatorname{tr} \mathcal{I}^{-1}(\theta) \ell_{n}^{\prime}(\theta)\left\{\ell_{n}^{\prime}(\theta)\right\}^{T} \stackrel{a . s .}{\rightarrow} \operatorname{tr}\left(\mathcal{I}^{-1}(\theta) \mathcal{I}(\theta)\right)=2,
$$

where, for any matrix $A, \operatorname{tr}(A)$ denotes trace of the matrix $A$.

Hence, combining the asymptotic inequalities we obtain that for $\theta \in \Theta_{n}^{c} \cap \Omega_{n}$, where $n$ is sufficiently 
large,

$$
\begin{aligned}
E_{\theta}\left(1-\Phi_{n}\right) & <e^{\alpha n \delta} \times e^{-2 \alpha n \mathcal{K}\left(\theta, \theta_{0}\right)} \times \sqrt{E_{\theta}\left(e^{2 \alpha \chi_{1}^{2}}\right) \times E_{\theta}\left(e^{4 \alpha \sqrt{2 \chi_{1}^{2}}}\right)} \\
& <e^{\alpha n \delta} \times e^{-2 \alpha n \delta} \times \sqrt{E_{\theta}\left(e^{2 \alpha \chi_{1}^{2}}\right) \times E_{\theta}\left(e^{4 \alpha \sqrt{2 \chi_{1}^{2}}}\right)} \\
& =e^{-\alpha \delta n} \times \sqrt{E_{\theta}\left(e^{2 \alpha \chi_{1}^{2}}\right) \times E_{\theta}\left(e^{4 \alpha \sqrt{2 \chi_{1}^{2}}}\right)} .
\end{aligned}
$$

For our choice of $\alpha$, the expectations in 3.40 are finite. Also since the right hand side of 3.40 does not depend upon $\theta$, (2)(ii) is proved in our case. That is, finally, posterior consistency (3.12) holds in our non-iid SDE set-up. The result can be summarized in the form of the following theorem.

Theorem 6 Assume the non-iid SDE set-up. Also assume conditions $\left(H 1^{\prime}\right)$ and $\left(H 3^{\prime}\right)-\left(H 10^{\prime}\right)$. For any $\delta>0$, let $\Theta_{\delta}=\left\{\left(\mu, \omega^{2}\right): \mathcal{K}\left(\theta, \theta_{0}\right)<\delta\right\}$, where $\mathcal{K}\left(\theta, \theta_{0}\right)$, defined as in $(3.6)$, is the proper KullbackLeibler divergence. Let the prior distribution $\pi$ of the parameter $\theta$ satisfy $\frac{d \pi}{d \nu}=h$ almost everywhere on $\Omega$, where $h(\theta)$ is any positive, continuous density on $\Omega$ with respect to the Lebesgue measure $\nu$. Then,

$$
\pi_{n}\left(\theta \in \Theta_{\delta}^{c} \mid X_{1}, \ldots, X_{n}\right) \rightarrow 0 \quad \text { a.s. }\left[P_{\theta_{0}}\right]
$$

\subsection{Asymptotic normality of the posterior distribution in the non- $i$ id set-up}

For asymptotic normality of the posterior in the iid situation, four regularity conditions, stated in Section 2.2.1 were necessary. In the non-iid framework, three more are necessary, in addition to the already presented four conditions. They are as follows (see Schervish (1995) for details).

\subsubsection{Extra regularity conditions in the non- $i i d$ set-up}

(5) The largest eigenvalue of $\Sigma_{n}$ goes to zero in probability.

(6) For $\delta>0$, define $\mathcal{N}_{0}(\delta)$ to be the open ball of radius $\delta$ around $\theta_{0}$. Let $\rho_{n}$ be the smallest eigenvalue of $\Sigma_{n}$. If $\mathcal{N}_{0}(\delta) \subseteq \Omega$, there exists $K(\delta)>0$ such that

$$
\lim _{n \rightarrow \infty} P_{\theta_{0}}\left(\sup _{\theta \in \Omega \backslash \mathcal{N}_{0}(\delta)} \rho_{n}\left[\ell_{n}(\theta)-\ell_{n}\left(\theta_{0}\right)\right]<-K(\delta)\right)=1 .
$$

(7) For each $\epsilon>0$, there exists $\delta(\epsilon)>0$ such that

$$
\lim _{n \rightarrow \infty} P_{\theta_{0}}\left(\sup _{\theta \in \mathcal{N}_{0}(\delta(\epsilon)),\|\gamma\|=1}\left|1+\gamma^{T} \Sigma_{n}^{\frac{1}{2}} \ell_{n}^{\prime \prime}(\theta) \Sigma_{n}^{\frac{1}{2}} \gamma\right|<\epsilon\right)=1 .
$$

In the non-iid case, the four regularity conditions presented in Section 2.2.1 and additional three provided above, are sufficient to guarantee 2.10 .

\subsubsection{Verification of the regularity conditions}

For $i=1,2$ and $j=1,2$, let the $(i, j)$-th element of $\ell_{n}^{\prime \prime}\left(\hat{\theta}_{n}\right)$ be denoted by $\ell_{n, i j}^{\prime \prime}\left(\hat{\theta}_{n}\right)$. Then $\ell_{n, i j}^{\prime \prime}\left(\hat{\theta}_{n}\right) / n$ admits the following Taylor's series expansion around $\theta_{0}$ :

$$
\frac{\ell_{n, i j}^{\prime \prime}\left(\hat{\theta}_{n}\right)}{n}=\frac{\ell_{n, i j}^{\prime \prime}\left(\theta_{0}\right)}{n}+\frac{\left(\hat{\theta}_{n}-\theta_{0}\right)^{T} \ell_{n, i j}^{\prime \prime \prime}\left(\theta_{n}^{*}\right)}{n}
$$


where $\theta_{n}^{*}$ lies between $\theta_{0}$ and $\hat{\theta}_{n}$. In the same way as 3.24 it can be easily shown that

$$
\frac{\ell_{n}^{\prime \prime}\left(\hat{\theta}_{n}\right)}{n} \stackrel{P}{\rightarrow}-\mathcal{I}\left(\theta_{0}\right)
$$

In other words, $-\ell_{n}^{\prime \prime}\left(\hat{\theta}_{n}\right)$ and $n \mathcal{I}\left(\theta_{0}\right)$ are asymptotically equivalent (in probability). Since the maximum eigenvalue of $n^{-1} \mathcal{I}^{-1}\left(\theta_{0}\right)$ goes to zero in probability as $n \rightarrow \infty$, so does the maximum eigenvalue of $\Sigma_{n}$. Hence, condition (5) holds.

To verify condition (6), note that again by Kolmogorov's strong law of large numbers,

$$
\frac{1}{n}\left(\ell_{n}(\theta)-\ell_{n}\left(\theta_{0}\right)\right) \stackrel{\text { a.s. }}{\rightarrow}-\mathcal{K}\left(\theta_{0}, \theta\right)
$$

where, $\mathcal{K}\left(\theta_{0}, \theta\right)$ is given by 3.5 , Now, writing $\rho_{n}\left[\ell_{n}(\theta)-\ell_{n}\left(\theta_{0}\right)\right]$ as $n \rho_{n}\left[\frac{\ell_{n}(\theta)-\ell_{n}\left(\theta_{0}\right)}{n}\right]$ and noting that $\Sigma_{n}=O_{P}\left(n^{-1}\right)$ implies $n \rho_{n} \stackrel{P}{\rightarrow} c$, where $c>0$, it follows from 3.46 that $\rho_{n}\left[\ell_{n}(\theta)-\ell_{n}\left(\theta_{0}\right)\right] \stackrel{P}{\rightarrow}$ $-c \mathcal{K}\left(\theta_{0}, \theta\right)<0$. Hence, condition (6) holds.

For condition (7) note that for $\theta \in \mathcal{N}_{0}(\delta(\epsilon)), \theta=\theta_{0}+\delta_{2} \frac{\theta_{0}}{\left\|\theta_{0}\right\|}$, where $0<\delta_{2} \leq \delta(\epsilon)$. So, using Taylor's series expansion around $\theta_{0}$, the $(i, j)$-th element of $\ell_{n}^{\prime \prime}(\theta) / n$ can be written as

$$
\frac{\ell_{n, i j}^{\prime \prime}(\theta)}{n}=\frac{\ell_{n, i j}^{\prime \prime}\left(\theta_{0}\right)}{n}+\delta_{2} \frac{\theta_{0}^{T} \ell_{n, i j}^{\prime \prime \prime}\left(\theta^{*}\right)}{n\left\|\theta_{0}\right\|},
$$

where $\theta^{*}$ lies between $\theta_{0}$ and $\theta$. As $n \rightarrow \infty, \frac{\ell_{n, i j}^{\prime \prime}\left(\theta_{0}\right)}{n}$ tends, in probability, to the $(i, j)$-th element of $-\mathcal{I}\left(\theta_{0}\right)$. Now notice that

$$
\frac{\left|\theta_{0}^{T} \ell_{n, i j}^{\prime \prime \prime}\left(\theta^{*}\right)\right|}{n\left\|\theta_{0}\right\|} \leq \frac{\left\|\ell_{n, i j}^{\prime \prime \prime}\left(\theta^{*}\right)\right\|}{n},
$$

so that $\frac{\left|\theta_{0}^{T} \ell_{n, i j}^{\prime \prime \prime}\left(\theta^{*}\right)\right|}{n\left\|\theta_{0}\right\|}=O_{P}(1)$ since $\frac{\left\|\ell_{n, i j}^{\prime \prime \prime}\left(\theta^{*}\right)\right\|}{n}=O_{P}(1)$ as before. Hence, it follows that $\ell_{n}^{\prime \prime}(\theta)=$ $O_{P}\left(-n \mathcal{I}\left(\theta_{0}\right)+n \delta_{2}\right)$. Since $\Sigma_{n}^{\frac{1}{2}}$ is asymptotically equivalent (in probability) to $n^{-\frac{1}{2}} \mathcal{I}^{-\frac{1}{2}}\left(\theta_{0}\right)$, condition (7) holds. We summarize our result in the form of the following theorem.

Theorem 7 Assume the non-iid set-up and conditions $\left(H 1^{\prime}\right)$ and $\left(H 3^{\prime}\right)-\left(H 9^{\prime}\right)$. Regarding $\left(H 3^{\prime}\right)$ we assume, in particular, that $\theta_{0} \in \operatorname{int}(\Omega)$. Let the prior distribution $\pi$ of the parameter $\theta$ satisfy $\frac{d \pi}{d \nu}=h$ almost everywhere on $\Omega$, where $h(\theta)$ is any density with respect to the Lebesgue measure $\nu$ which is positive and continuous at $\theta_{0}$. Then, letting $\Psi_{n}=\Sigma_{n}^{-1 / 2}\left(\theta-\hat{\theta}_{n}\right)$, for each compact subset $B$ of $\mathbb{R} \times \mathbb{R}^{+}$and each $\epsilon>0$, the following holds:

$$
\lim _{n \rightarrow \infty} P_{\theta_{0}}\left(\sup _{\Psi_{n} \in B}\left|\pi_{n}\left(\Psi_{n} \mid X_{1}, \ldots, X_{n}\right)-\xi\left(\Psi_{n}\right)\right|>\epsilon\right)=0 .
$$

\section{Summary and discussion}

In this paper, we have investigated Bayesian posterior consistency in the context of $S D E$ 's consisting of drift terms depending linearly upon random effect parameters. In particular, we have proved posterior consistency and asymptotic normality in both $i i d$ and non-iid set-ups, as the number of observed processes tends to infinity. In Section S-1 of our supplement we have illustrated our results with concrete examples, showing when posterior consistency will hold and not hold. Even in the dependent set-up we have illustrated, with examples, when consistency will hold and fail; see Section S-1.2 of the supplement. The latter examples can be looked upon as providing insights into the Bayesian asymptotic theory of dependent sets of $S D E$ 's. 
It is also important to illustrate the value of Bayesian analysis in $S D E$-based random effects model, particularly because, as per our results, at least asymptotically Bayesian analysis does not have edge over its classical counterpart. However, in small samples, Bayesian analysis can outperform classical analysis when adequate prior knowledge on the parameter in question is available. In Section S-2 of the supplement we present a simulation study to illustrate the advantage of Bayesian analysis in small samples. For realistic practical applications we include a brief discussion on elicitation of prior information and posterior computations in Section S-3 of the supplement.

Since discretization of the underlying continuous time processes is important for inference in practical situations, it is worth providing some remarks regarding discretization in our Bayesian context. Firstly, note that discretized version of $U_{i}$ and $V_{i}$, as provided in Delattre et al. (2013), are given by

$$
\begin{aligned}
U_{i}^{m} & =\sum_{k=0}^{m-1} \frac{b\left(X_{i}\left(t_{k}\right)\right)}{\sigma^{2}\left(X_{i}\left(t_{k}\right)\right)}\left(X_{i}\left(t_{k+1}\right)-X_{i}\left(t_{k}\right)\right), \\
V_{i}^{m} & =\sum_{k=0}^{m-1} \frac{b^{2}\left(X_{i}\left(t_{k}\right)\right)}{\sigma^{2}\left(X_{i}\left(t_{k}\right)\right)}\left(t_{k+1}-t_{k}\right) .
\end{aligned}
$$

Under mild conditions, Lemma 3 of Delattre et al. (2013) provides bounds for the expectation associated with the differences $U_{i}-U_{i}^{m}$ and $V_{i}-V_{i}^{m}$. We utilize the result to deduce that as $m \rightarrow \infty$, $\pi_{n}\left(\cdot \mid X_{1}, \ldots, X_{n}\right)-\pi_{n}^{(m)}\left(\cdot \mid X_{1}, \ldots, X_{n}\right)=o_{P_{0}}(1)$, where $\pi_{n}^{(m)}\left(\cdot \mid X_{1}, \ldots, X_{n}\right)$ is the posterior density associated with $\left(U_{i}^{m}, V_{i}^{m}\right) ; i=1, \ldots, n$. Thus, it is not difficult to see that our consistency and asymptotic normality results for both iid and non-iid cases continue to hold as $m \rightarrow \infty, n \rightarrow \infty$ such that $\frac{m}{n} \rightarrow \infty$.

Finally, it is important to remark that in this paper we have confined ourselves to one-dimensional random effect parameters and one-dimensional $S D E$ 's. Although our non-iid $S D E$ framework admits straightforward generalization to multi-dimensional situations (for the iid counterpart multivariate generalization has been considered by Delattre et al. (2013)), generalization of our asymptotic theory to high dimensions does not seem to be as straightforward. We reserve this problem for our future research.

\section{Acknowledgments}

Sincere gratitude goes to three anonymous reviewers whose detailed comments have led to much improved presentation of our article. The first author gratefully acknowledges her CSIR Fellowship, Govt. of India. 


\section{Supplementary Material}

\section{S-1 Examples illustrating consistency and inconsistency}

In this section we consider simple examples in our $S D E$ set-up for illustrating consistency and inconsistency in simple terms. For simplicity, in all the examples we consider the set of $S D E$ 's having the following form: for $i=1, \ldots, n$,

$$
d X_{i}(t)=\phi_{i} d t+d W_{i}(t)
$$

that is, we set $b(\cdot)=\sigma(\cdot) \equiv 1$. Hence, for $i=1, \ldots, n$,

$$
U_{i}=\phi_{i} T_{i}+W_{i}\left(T_{i}\right) \text { and } V_{i}=T_{i}
$$

\section{S-1.1 Example 1}

We assume that for $i=1, \ldots, n, \phi_{i} \stackrel{i i d}{\sim} N(\mu, 1)$, that is, we set $\omega^{2}=1$. Letting $\mu_{0}$ be the true value of $\mu$, we investigate consistency of the posterior of $\mu$. Closed form expression of the posterior is available if we put $\pi(\mu) \equiv N\left(A, B^{2}\right)$ prior on $\mu$; here $-\infty<A<\infty$ and $B>0$. Indeed, $\pi_{n}\left(\mu \mid X_{1}, \ldots, X_{n}\right) \equiv N\left(\hat{\mu}_{n}, \hat{\sigma}_{n}^{2}\right)$, where

$$
\begin{aligned}
\hat{\mu}_{n} & =\frac{\sum_{i=1}^{n} \frac{U_{i}}{1+V_{i}}+\frac{A}{B^{2}}}{\sum_{i=1}^{n} \frac{V_{i}}{1+V_{i}}+\frac{1}{B^{2}}} \\
\hat{\sigma}_{n}^{2} & =\frac{1}{\sum_{i=1}^{n} \frac{V_{i}}{1+V_{i}}+\frac{1}{B^{2}}}=\frac{1}{\sum_{i=1}^{n} \frac{T_{i}}{1+T_{i}}+\frac{1}{B^{2}}} .
\end{aligned}
$$

Note that $\hat{\mu}_{n}$ follows the normal distribution with mean and variance given by the following:

$$
\begin{aligned}
E\left(\hat{\mu}_{n}\right) & =\frac{\mu_{0} \sum_{i=1}^{n} \frac{T_{i}}{1+T_{i}}+\frac{A}{B^{2}}}{\sum_{i=1}^{n} \frac{T_{i}}{1+T_{i}}+\frac{1}{B^{2}}} ; \\
\operatorname{Var}\left(\hat{\mu}_{n}\right) & =\frac{\sum_{i=1}^{n} \frac{T_{i}}{1+T_{i}}}{\left(\sum_{i=1}^{n} \frac{T_{i}}{1+T_{i}}+\frac{1}{B^{2}}\right)^{2}} .
\end{aligned}
$$

Now, for any $\epsilon>0$, by Chebychev's inequality,

$$
P\left(\left|\hat{\mu}_{n}-\mu_{0}\right|>\epsilon\right)<\epsilon^{-4} E\left(\hat{\mu}_{n}-\mu_{0}\right)^{4}=O\left\{\left(\operatorname{Var}\left(\hat{\mu}_{n}\right)\right)^{2}\right\}=O\left\{\left(\frac{\sum_{i=1}^{n} \frac{T_{i}}{1+T_{i}}}{\left(\sum_{i=1}^{n} \frac{T_{i}}{1+T_{i}}+\frac{1}{B^{2}}\right)^{2}}\right)^{2}\right\} .
$$

\section{S-1.1.1 Case 1: $T_{i}=T$ for all $i \geq 1$}

In this case, $\operatorname{Var}\left(\hat{\mu}_{n}\right)=O\left(n^{-1}\right)$. Hence, it follows from $(\mathrm{S}-1.7)$ that for any $\epsilon>0$,

$$
\sum_{n=1}^{\infty} P\left(\left|\hat{\mu}_{n}-\mu_{0}\right|>\epsilon\right)<\infty
$$

so that $\hat{\mu}_{n} \stackrel{a . s .}{\rightarrow} \mu_{0}$. Moreover, since $\hat{\sigma}_{n}^{2} \rightarrow 0$, consistency of the posterior of $\mu$, for almost all data sequences, follows. 


\section{S-1.1.2 Case 2: $T_{i}$ different and $\sum_{i=1}^{\infty} \frac{T_{i}}{1+T_{i}}=\infty$}

A typical instance of $T_{i}$ for which $\sum_{i=1}^{\infty} \frac{T_{i}}{1+T_{i}}=\infty$ is $\frac{T_{i}}{1+T_{i}}=\frac{1}{i}$ (so that $T_{i}=\frac{1}{i-1}$ ), for $i>1 ; T_{1}=c_{0}$, say, for any constant $c_{0}$. For $\sum_{i=1}^{\infty} \frac{T_{i}}{1+T_{i}}=\infty, \hat{\sigma}_{n}^{2} \rightarrow 0$. Also, $E\left(\hat{\mu}_{n}\right) \rightarrow \mu_{0}$ and $\operatorname{Var}\left(\hat{\mu}_{n}\right) \rightarrow 0$, implying that $\hat{\mu}_{n} \stackrel{P}{\rightarrow} \mu_{0}$. In fact, since $\sum_{i=1}^{n} \frac{T_{i}}{1+T_{i}}=O(n)$ as $n \rightarrow \infty$, it follows from $(\mathrm{S}-1.7)$ that for any $\epsilon>0$,

$$
\sum_{n=1}^{\infty} P\left(\left|\hat{\mu}_{n}-\mu_{0}\right|>\epsilon\right)<\infty
$$

Hence, consistency of the posterior of $\mu$ for almost all data sequences, follows. Thus, in this situation, for the sequence $T_{i}=\frac{1}{i-1}$ for $i>1 ; T_{1}=c_{0}$ belonging to some compact $\mathfrak{T}$ is appropriate.

Now observe that if $T_{i} \rightarrow \infty$ as $i \rightarrow \infty$, then also $\sum_{i=1}^{\infty} \frac{T_{i}}{1+T_{i}}=\infty$, enforcing consistency. Clearly in this case $\mathfrak{T}$ is non-compact. This example shows that compactness is sufficient, but not necessary for consistency.

S-1.1.3 Case 3: $\sum_{i=1}^{\infty} \frac{T_{i}}{1+T_{i}}<\infty$

A typical instance of $T_{i}$ for which $\sum_{i=1}^{\infty} \frac{T_{i}}{1+T_{i}}<\infty$ is $\frac{T_{i}}{1+T_{i}}=\frac{1}{i^{2}}$ (so that $T_{i}=\frac{1}{i^{2}-1}$ ), for $i>1 ; T_{1}=c_{0}$, say, for any constant $c_{0}$.

In such cases, $\hat{\sigma}_{n}^{2} \nrightarrow 0$, showing that the posterior of $\mu$ is inconsistent. In other words, even though the sequence $T_{i}=\frac{1}{i^{2}-1}$ for $i>1 ; T_{1}=c_{0}$ belongs to some compact $\mathfrak{T}$, consistency still does not hold. This example shows that not all convergent sequences in compact $\mathfrak{T}$ ensure consistency. As per our theory, we can only assert that there exists at least one convergent subsequence of any sequence in $\mathfrak{T}$ for which consistency is attained.

\section{S-1.2 Example 2}

In Example 1 we considered the set-up where, for $i=1, \ldots, n, \phi_{i} \stackrel{i i d}{\sim} N(\mu, 1)$. This choice makes the $S D E$ 's independent. Indeed, in this paper, we have provided theoretical results assuming that the $S D E$ 's are at least independent. However, at this point, we investigate with simulations, when posterior consistency holds and fails, assuming a dependent set-up, the dependence induced by the following $n$-variate normal distribution of $\phi_{n}=\left(\phi_{1}, \ldots, \phi_{n}\right)$ :

$$
\phi_{n} \sim N_{n}\left(\mu \mathbf{1}_{n}, \omega^{2} \boldsymbol{\Sigma}_{n}\right)
$$

where $\mathbf{1}_{n}$ is the $n$-component vector with all entries $1, \omega>0$, and $\boldsymbol{\Sigma}_{n}$ is an $n \times n$ covariance matrix. Let $\boldsymbol{U}=\left(U_{1}, \ldots, U_{n}\right)^{\prime}$, and $\boldsymbol{V}=\operatorname{diag}\left\{V_{1}, \ldots, V_{n}\right\}$, that is, $\boldsymbol{V}$ is an $n \times n$ diagonal matrix with the $i$-th diagonal element $V_{i}$. Assuming that $\pi(\mu) \equiv N\left(A, B^{2}\right)$, it follows that the posterior of $\mu$ is given by

$$
\pi_{n}\left(\mu \mid X_{1}, \ldots, X_{n}\right) \equiv N\left(\hat{\mu}_{n}, \hat{\sigma}_{n}^{2}\right),
$$

where

$$
\begin{aligned}
& \hat{\mu}_{n}=\frac{\omega^{2} \mathbf{1}^{\prime} \boldsymbol{\Sigma}_{n}^{-1}\left(\omega^{2} \boldsymbol{V}+\boldsymbol{\Sigma}_{n}^{-1}\right) \boldsymbol{U}+\frac{A}{B^{2}}}{\mathbf{1}^{\prime} \boldsymbol{\Sigma}_{n}^{-1}\left\{\boldsymbol{\Sigma}_{n}-\left(\omega^{2} \boldsymbol{V}+\boldsymbol{\Sigma}_{n}^{-1}\right)^{-1}\right\} \boldsymbol{\Sigma}_{n}^{-1} \mathbf{1}+\frac{1}{B^{2}}} ; \\
& \hat{\sigma}_{n}^{2}=\frac{\omega^{2}}{\mathbf{1}^{\prime} \boldsymbol{\Sigma}_{n}^{-1}\left\{\boldsymbol{\Sigma}_{n}-\left(\omega^{2} \boldsymbol{V}+\boldsymbol{\Sigma}_{n}^{-1}\right)^{-1}\right\} \boldsymbol{\Sigma}_{n}^{-1} \mathbf{1}+\frac{1}{B^{2}}} .
\end{aligned}
$$

We conduct two simulation studies to investigate consistency in this set-up. 


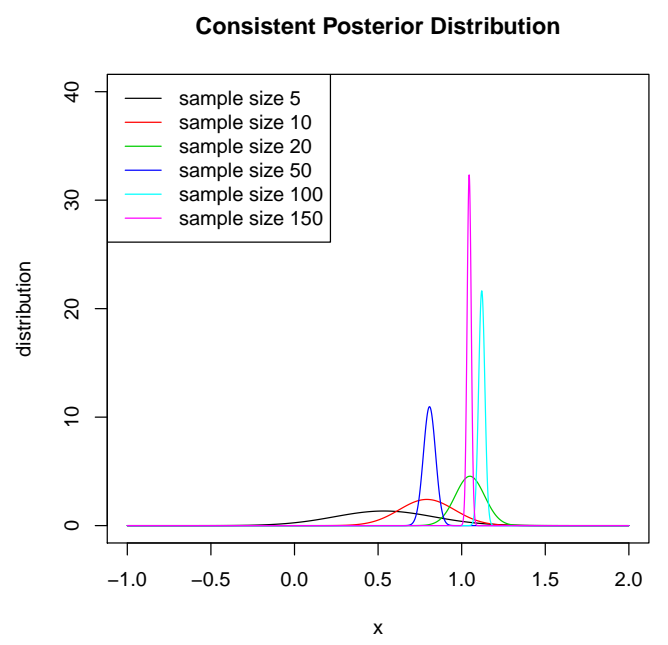

Figure S-1: Illustration of posterior consistency in the weakly dependent set-up.

\section{S-1.2.1 First simulation study - weak dependence structure}

As in Example 1, we set $b(\cdot)=\sigma(\cdot) \equiv 1$ in our $S D E$ 's, so that $U_{i}=\phi_{i} T_{i}+W_{i}\left(T_{i}\right)$ and $V_{i}=T_{i}$; we set $T_{i}=5$ for each $i$. For the distribution of $\phi_{n}$ of the form $(\mathrm{S}-1.8)$ we set the true value $\mu_{0}=1$. Also, for simplicity, we set $\omega^{2}=1$. For the covariance matrix $\boldsymbol{\Sigma}_{n}$, we consider the following weakly dependent structure:

$$
\boldsymbol{\Sigma}_{n}=\left(\begin{array}{ccccccc}
1 & \frac{1}{3} & 0 & 0 & 0 & \cdots & 0 \\
\frac{1}{3} & 1 & \frac{1}{3} & 0 & 0 & \cdots & 0 \\
0 & \frac{1}{3} & 1 & \frac{1}{3} & 0 & \cdots & 0 \\
\vdots & \vdots & \vdots & \vdots & \vdots & \vdots & \vdots \\
0 & 0 & 0 & \cdots & 0 & \frac{1}{3} & 1
\end{array}\right) .
$$

For any $n$, this matrix is strictly diagonally dominant, and hence positive definite.

We generate the data by generating $\phi_{n}$ from the $n$-variate normal $(\mathrm{S}-1.8)$, generating $W_{i}\left(T_{i}\right)$ and then forming $U_{i}$ and $V_{i}$, for $i=1, \ldots, n$.

Figure S-1 displays the posterior distribution of $\mu$ for various sample sizes. Clearly, for larger sample sizes, the posterior increasingly concentrates around the true value $\mu_{0}=1$, thus demonstrating posterior consistency.

\section{S-1.2.2 Second simulation study - strong dependence structure}

In the second experiment, we consider a strong dependence structure between the components of $\phi_{n}$, quantified by

$$
\boldsymbol{\Sigma}_{n}=\left(\begin{array}{ccccccc}
1 & \frac{1}{3} & \frac{1}{3} & \frac{1}{3} & \frac{1}{3} & \cdots & \frac{1}{3} \\
\frac{1}{3} & 1 & \frac{1}{3} & \frac{1}{3} & \frac{1}{3} & \cdots & \frac{1}{3} \\
\frac{1}{3} & \frac{1}{3} & 1 & \frac{1}{3} & \frac{1}{3} & \cdots & \frac{1}{3} \\
\vdots & \vdots & \vdots & \vdots & \vdots & \vdots & \vdots \\
\frac{1}{3} & \frac{1}{3} & \frac{1}{3} & \cdots & \frac{1}{3} & \frac{1}{3} & 1
\end{array}\right) .
$$

The rest of the set-up remains exactly same as in the previous experiment with the weak dependence structure.

Figure $\mathrm{S}$-2 displays the posterior distribution of $\mu$ for various sample sizes, in the strongly dependent situation. Note that even for large sample sizes, the variability of the posterior distribution does not decrease, which is clearly indicative of posterior inconsistency. Hence, in the strongly dependent $S D E$ set-up, posterior consistency does not hold. 


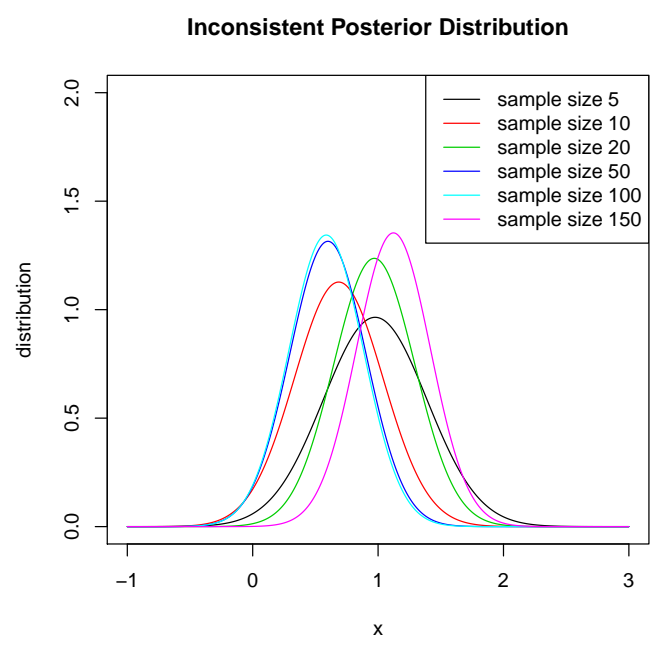

Figure S-2: Illustration of posterior inconsistency in the strongly dependent set-up.

\section{S-2 Illustration of advantages of Bayesian analysis over classical infer- ence in small samples}

It is important to illustrate the value of Bayesian analysis in $S D E$-based random effects model, particularly because, as per our results, at least asymptotically Bayesian analysis does not have edge over its classical counterpart. However, in small samples, Bayesian analysis can outperform classical analysis when adequate prior knowledge on the parameter in question is available. We undertake a simulation study to illustrate the advantage of Bayesian analysis in small samples. Briefly, we consider the same set-up as Section S-1, assuming that the true value $\mu_{0}=1$. We choose the prior $\pi(\mu) \equiv N\left(A, B^{2}\right)$ with $A=0$ and $B=1.5$. In this set-up, we obtain the $95 \%$ confidence interval in the classical case and $95 \%$ highest posterior density (HPD) interval in the Bayesian case, for the true parameter $\mu_{0}$. The results are presented in Table $\mathrm{S}-1$. Note that all the intervals, for both classical and Bayesian analyses include the true value $\mu_{0}=1$, however, the lengths of the Bayesian 95\% HPD intervals are significantly shorter than the corresponding 95\% classical confidence intervals in all the cases. In fact, lesser the sample size, larger is the difference between the lengths of the Bayesian and classical intervals. In real situations involving random effects, adequately informative prior opinions regarding the parameter in question can often be obtained, and this small example demonstrates that such information can substantially enhance Bayesian inference.

Table S-1: Comparison between classical and Bayesian analysis of $S D E$-based random effects model for small samples.

\begin{tabular}{|c||c|c||c|c|}
\hline $\begin{array}{c}\text { Sample } \\
\text { Size }\end{array}$ & $\begin{array}{c}\text { Classical 95\% Confidence } \\
\text { Interval }\end{array}$ & $\begin{array}{c}\text { Interval } \\
\text { Length }\end{array}$ & $\begin{array}{c}\text { Bayes 95\% HPD } \\
\text { Interval }\end{array}$ & $\begin{array}{c}\text { Interval } \\
\text { Length }\end{array}$ \\
\hline 2 & $(-1.052,3.243)$ & 4.294 & $(-0.934,1.866)$ & 2.801 \\
3 & $(-0.744,2.293)$ & 3.036 & $(-0.716,1.681)$ & 2.397 \\
4 & $(-0.607,1.872)$ & 2.479 & $(-0.597,1.508)$ & 2.105 \\
5 & $(-0.526,1.621)$ & 2.147 & $(-0.521,1.374)$ & 1.894 \\
6 & $(-0.470,1.450)$ & 1.920 & $(-0.468,1.268)$ & 1.735 \\
7 & $(-0.429,1.324)$ & 1.753 & $(-0.428,1.182)$ & 1.610 \\
8 & $(-0.397,1.226)$ & 1.623 & $(-0.397,1.112)$ & 1.508 \\
9 & $(-0.372,1.146)$ & 1.518 & $(-0.371,1.052)$ & 1.423 \\
10 & $(-0.351,1.081)$ & 1.431 & $(-0.350,1.001)$ & 1.351 \\
\hline
\end{tabular}




\section{S-3 Brief discussion on prior elicitation and posterior computation in re- alistic situations}

In our simulation studies, for simplicity of illustrations, we have considered the $N\left(A, B^{2}\right)$ prior on $\mu$, assuming $A$ and $B$ to be known; also we have set $\omega^{2}=1$. In realistic situations elicitation of such strong prior information is not always straightforward. Moreover, the random effects parameters $\phi_{i}$ may be $d$-dimensional, so that $\mu$ is to be replaced by the $d$-dimensional vector $\boldsymbol{\mu}=\left(\mu_{1}, \ldots, \mu_{d}\right)$, and $\omega^{2}$ needs to be replaced with the $d \times d$ matrix $\boldsymbol{\Sigma}$. The multidimensional situation makes appropriate choices of priors even more difficult. In the context of $S D E$-based pharmacokinetic models Yan et al. (2014), following de la Cruz-Mesía and Marshall (2006), proposed independent normal priors for the components of $\boldsymbol{\mu}$, given by $\mu_{i} \sim N\left(A_{i}, B_{i}^{2}\right)$, and an inverse Wishart prior for $\boldsymbol{\Sigma}$ with scale matrix $\boldsymbol{\Sigma}_{0}$ and degrees of freedom $d+1$. However, because of the difficulties of eliciting information, they assume non-informative priors for the hyperparameters.

Informative priors can be elicited if historical data, that is, data associated with previous studies, are available. Then, following Yan et al. (2014), using the aforementioned non-informative priors, one can first obtain the posterior distributions of $\boldsymbol{\mu}$ and $\boldsymbol{\Sigma}$, given only the historical data. These posterior distributions based on historical data can then be used as informative prior distributions for Bayesian analysis of the current data. Such an approach has been advocated and formalized in the context of generalized linear mixed models by Ibrahim and Chen (2000).

It is important to remark that for complicated priors as discussed above, the posterior distribution need not be available in closed form. Even obtaining closed form of the prior, which is the posterior given the historical data, is not guaranteed. In the context of generalized linear mixed effects model, Ibrahim and Chen (2000) propose a Gibbs sampling algorithm based on the centering strategies of Gelfand et al. (1996), for efficiently sampling from the desired posterior, given the current data. However, since our $S D E$-based model does not fall within the class of generalized linear mixed models, such Gibbs sampling strategies need not be available in our case. Instead, the Metropolis-Hastings (MH) method can be used to sample from the posterior. In the context of pharmacokinetic models, Yan et al. (2014) estimate the parameters using a combination of extended Kalman filter and random walk MH algorithm; see also Donnet and Samson (2013) for a comprehensive review on various techniques of classical and Bayesian estimation of $S D E$ 's for pharmacokinetic/pharmacodynamic models.

For a fully Bayesian approach, required posterior computations can be carried out using only the $\mathrm{MH}$ methodology. The MH methodology, however, has drawbacks in that convergence can often be quite slow and computations can be very burdensome, when the dimension $d$ is large. To bypass these problems, Dutta and Bhattacharya (2014) have developed a novel methodology which they refer to as Transformation based Markov Chain Monte Carlo (TMCMC), that can update the entire high-dimensional parameter set in a single block using simple deterministic transformations of a single random variable, thus effectively reducing the multidimensional parameter to a single dimension. Apart from drastically reducing computing time, the method promises much improved acceptance rates and convergence properties; see Dutta and Bhattacharya (2014), Dey and Bhattacharya (2015a), Dey and Bhattacharya (2015b), Dey and Bhattacharya (2016).

\section{References}

Bennett, J. E., Racine-Poon, A., and Wakefield, J. C. (1996). MCMC for Nonlinear Hierarchical Models. In W. R. Gilks, S. Richardson, and D. J. Spiegelhalter, editors, Markov Chain Monte Carlo in Practice, pages 339-350, London. Chapman \& Hall.

Choi, T. and Schervish, M. J. (2007). On Posterior Consistency in Nonparametric Regression Problems. Journal of Multivariate Analysis, 98, 1969-1987.

de la Cruz-Mesía, R. and Marshall, G. (2006). Non-Linear Random Effects Models With Continuous Time Autoregressive Errors: A Bayesian Approach. Statistics in Medicine, 25, 1471-1484. 
Delattre, M., Genon-Catalot, V., and Samson, A. (2013). Maximum Likelihood Estimation for Stochastic Differential Equations with Random Effects. Scandinavian Journal of Statistics, 40, 322-343.

Dey, K. K. and Bhattacharya, S. (2015a). On Optimal Scaling of Additive Transformation based Markov Chain Monte Carlo. Submitted. Available at "http://arxiv.org/abs/1307.1446".

Dey, K. K. and Bhattacharya, S. (2015b). On Optimal Scaling of Additive Transformation based Markov Chain Monte Carlo Under Non-Regular Cases. Submitted. Available at "http://arxiv.org/abs/1405.0913".

Dey, K. K. and Bhattacharya, S. (2016). On Geometric Ergodicity of Additive and Multiplicative Transformation based Markov Chain Monte Carlo in High Dimensions. Brazilian Journal of Probability and Statistics. To appear. Available at "http://arxiv.org/pdf/1312.0915".

Donnet, S. and Samson, A. (2013). A Review on Estimation of Stochastic Differential Equations for Pharmacokinetic/Pharmacodynamic Models. In Advanced Drug Delivery Reviews, pages 1-25. Elsevier. pp. $1<10.1018 /$ j.addr.2013.03.005 $>$. $<$ hal-00777774 $>$.

Dutta, S. and Bhattacharya, S. (2014). Markov Chain Monte Carlo Based on Deterministic Transformations. Statistical Methodology, 16, 100-116. Also available at http://arxiv.org/abs/1106.5850. Supplement available at http://arxiv.org/abs/1306.6684.

Gelfand, A. E., Sahu, S. K., and Carlin, B. P. (1996). Efficient Parametrisations for Generalized Linear Mixed Models (with discussion). In J. M. Bernardo, J. O. Berger, A. P. Dawid, and A. F. M. Smith, editors, Bayesian Statistics 5, pages 165-180, Oxford, UK. Oxford University Press.

Hoadley, B. (1971). Asymptotic Properties of Maximum Likelihood Estimators for the Independent not Identically Distributed Case. The Annals of Mathematical Statistics, 42, 1977-1991.

Ibrahim, J. G. and Chen, M.-H. (2000). Prior Elicitation and Variable Selection for Generalized Linear Mixed Models. In D. K. Dey, S. K. Ghosh, and B. K. Mallick, editors, Generalized Linear Models: A Bayesian Perspective, pages 41-53, New York. Marcel Dekker, Inc.

Maitra, T. and Bhattacharya, S. (2015). Supplement to"On Bayesian Asymptotics in Stochastic Differential Equations with Random Effects".

Maitra, T. and Bhattacharya, S. (2016). On Asymptotics Related to Classical Inference in Stochastic Differential Equations with Random Effects. Statistics and Probability Letters, 110, 278-288. Also available at "http://arxiv.org/abs/1407.3968".

Schervish, M. J. (1995). Theory of Statistics. Springer-Verlag, New York.

Serfling, R. J. (1980). Approximation Theorems of Mathematical Statistics. John Wiley \& Sons, Inc., New York.

Wakefield, J. C. (1996). The Bayesian Analysis of Population Phamacokinetic Models. Journal of the American Statistical Association, 91, 62-75.

Wakefield, J. C., Smith, A. F. M., Racine-Poon, A., and Gelfand, A. E. (1994). Bayesian Analysis of Linear and Nonlinear Population Models by Using the Gibbs Sampler. Journal of the Royal Statistical Society. Series C (Applied Statistics), 43, 201-221.

Yan, F.-R., Zhang, P., Liu, J.-L., Tao, Y.-X., Lin, X., Lu, T., and Lin, J.-G. (2014). Parameter Estimation of Population Pharmacokinetic Models with Stochastic Differential Equations: Implementation of an Estimation Algorithm. Journal of Probability and Statistics, 2014, 1-8. 